\title{
The Contest Between the Kernels in the Selberg Trace Formula for the $(q+1)$-regular Tree
}

\author{
Matthew D. Horton, Derek B. Newland, and Audrey A. Terras \\ AbStRact. We compare half a dozen kernels in the regular tree analogue of \\ Selberg's trace formula and the deductions about finite $(q+1)$-regular graphs \\ $\mathrm{X}$ that result. Included are the following kernels: \\ 1) the kernel given by the indicator function of the set of points in the \\ tree at a fixed distance from the origin, \\ 2) the (ubiquitous) heat kernel, \\ 3) the kernel used to prove that the Ihara zeta function of $\mathrm{X}$ is the recip- \\ rocal of a polynomial, \\ 4) the kernel whose spherical transform is the indicator function of an \\ interval on the real line, \\ $5)$ the resolvent kernel or Green's function, \\ 6) the kernel whose horocycle transform is the indicator function of the \\ set $\{-n, n\}$.
}

\section{Introduction}

Suppose $\mathbb{T}_{k}$ denotes the $k=(q+1)$-regular tree. This means $\mathbb{T}_{k}$ is a connected graph with no cycles such that each vertex has degree $k=q+1$. See Figure 1. By a kernel (not to be confused with the concept from algebra) we mean a function $g: \mathbb{T}_{k} \times \mathbb{T}_{k} \longrightarrow \mathbb{C}$ to be used in the analogue of an integral operator $L_{g}$ on functions $\varphi: \mathbb{T}_{k} \longrightarrow \mathbb{C}$, defined by

$$
L_{g} \varphi(x)=\sum_{y \in \mathbb{T}_{k}} g(x, y) \varphi(y) .
$$

Since $\mathbb{T}_{k}$ is infinite, we will have to worry about convergence unless we view everything as a generalized function or distribution.

We will be taking the trace of $L_{g}$ restricted to functions $f: \Gamma \backslash \mathbb{T}_{k} \longrightarrow \mathbb{C}$, where $\Gamma$ is the fundamental group of a finite $k$-regular graph $X$ which can be identified with $\Gamma \backslash \mathbb{T}_{k}$. This quotient, is viewed as a finite analog of a compact Riemann surface. Our trace formula is thus analogous to Selberg's original trace formula (see [34]). See Terras $[\mathbf{4 2}]$ or the Terras website

$$
\text { http : //www.math.ucsd.edu/ aterras/newchaos.pdf }
$$

for tables comparing three types of trace formulas. A reference for the continuous trace formula is Terras [40].

2000 Mathematics Subject Classification. Primary 11F72; Secondary 05C50.

Key words and phrases. Selberg trace formula, Heat Kernel, k-regular tree. 


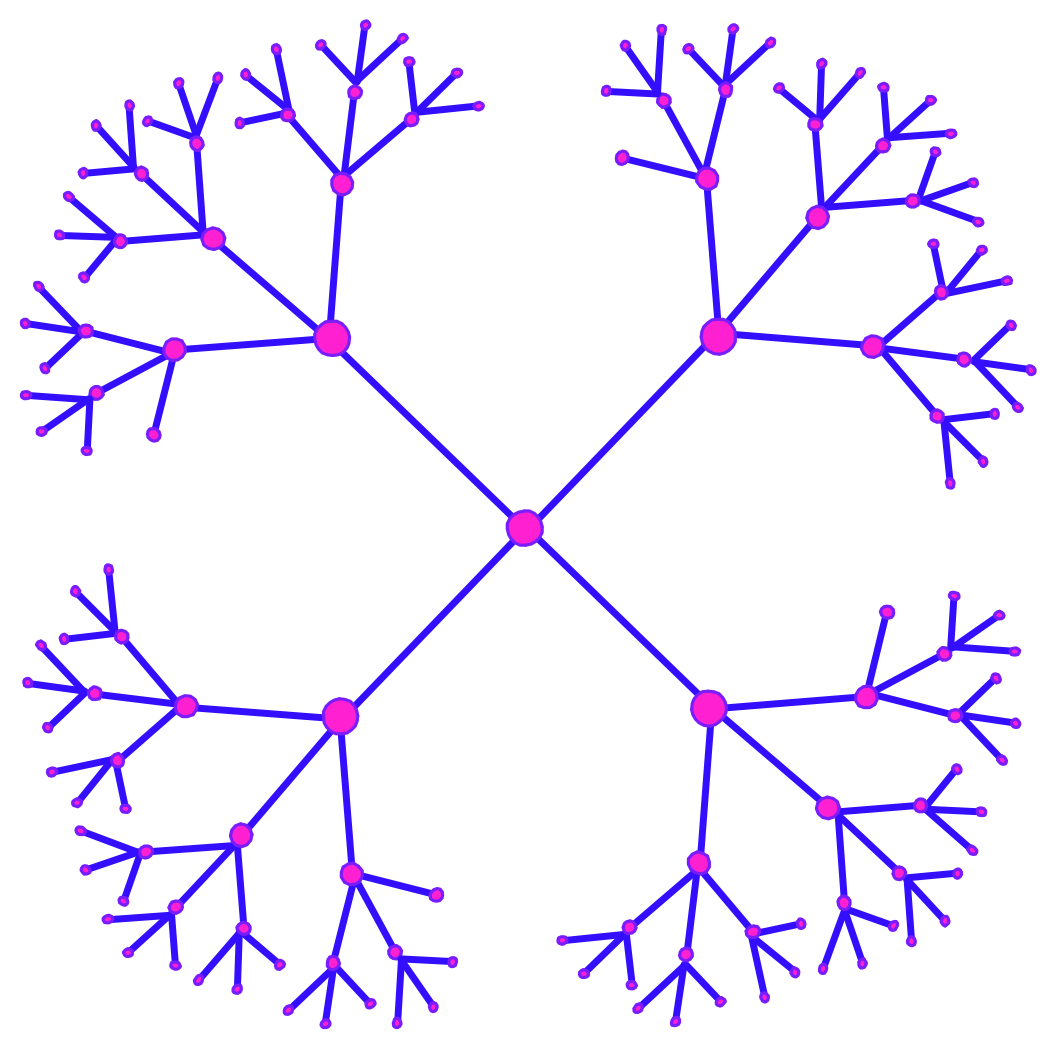

FiguRE 1. $\mathbb{T}_{4}=4$-regular tree (i.e., connected 4-regular graph with no cycles).

1.1. The Six Kernels. Our goal is to compare six kernels to see which is more informative about the finite $k$-regular graph $X$. Some of the kernels (e.g., the heat kernel) have classical counterparts and others do not.

Definition 1. For $x, y \in \mathbb{T}_{k}$, define the distance between $x$ and $y$ by $d(x, y)=$ number of edges in the unique path in $\mathbb{T}_{k}$ connecting $x$ and $y$.

Our kernels $g$ will have the form $g(x, y)=g(d(x, y))$, for $x, y \in \mathbb{T}_{k}$.

Kernel \#1) The kernel $f_{n}$ is defined to be

$$
f_{n}(x, y)= \begin{cases}1, & \text { if } d(x, y)=n \\ 0, & \text { otherwise }\end{cases}
$$

Kernel \# 2) The heat kernel $u_{t}$ is that whose spherical transform (see Definition 19) is

$$
\widehat{u_{t}}(\lambda)=e^{\frac{1}{k}(\lambda-k) t} .
$$

Kernel \#3) The kernel $\alpha_{u}$ is the one used to show that the reciprocal of the Ihara zeta function (see Definition 8 ) of $X$ is a polynomial. 
Kernel \#4) The kernel $f_{\alpha, \beta}$ is that with spherical transform (see Definition 19)

$$
\widehat{f_{\alpha, \beta}}(\lambda)= \begin{cases}1, & \text { if } \alpha \leq \lambda \leq \beta \\ 0, & \text { otherwise. }\end{cases}
$$

Kernel \#5) The resolvent kernel or Green's function $G_{w}$ is defined by having spherical transform

$$
\widehat{G_{w}}(\lambda)=\frac{1}{\lambda-\lambda(w)}, \text { where } \lambda(w)=q^{w}+q^{1-w} \neq \lambda(s) .
$$

Kernel \#6) The kernel $g_{n}$ is that whose horocycle transform (see Definition 17) is

$$
H g_{n}(a)= \begin{cases}1, & \text { if } a= \pm n \\ 0, & \text { otherwise }\end{cases}
$$

\subsection{Consequences of the Selberg Trace Formula} for the Six Kernels. Let us next give a list of results derived from the trace formula applied to the kernels. First we need some definitions from graph theory. For more graph theory basics, see Biggs [5] or Bollobás [6]. We consider a finite $k$-regular graph $X$ with its edges oriented arbitrarily. Denote the edges $e_{1}, \ldots, e_{m}, e_{m+1}=e_{1}^{-1}, \ldots, e_{2 m}=e_{m}^{-1}$, where the inverse of an oriented edge is that edge with the opposite orientation. A closed path $C$ in $X$ has the form $C=a_{1} \cdots a_{s}$, where $a_{j}$ denotes an oriented edge of $X$. The path $C$ has backtracking if $a_{j+1}=a_{j}^{-1}$, for some $j$ and the path $C$ has a tail if $a_{s}=a_{1}^{-1}$.

Definition 2. A prime (or primitive) path $C$ in $X$ means that $C$ is a closed, backtrackless, tailless path in $X$ such that $C \neq D^{n}, n>1$.

Definition 3. The set of all paths obtained from $C$ by changing the starting vertex is denoted $[C]$. If $C$ is a prime path, we say $[C]$ is a prime.

Definition 4. The length of a path $C=a_{1} \cdots a_{s}$ is the number of edges in $C$ and is denoted $\nu(C)=s$.

There are two numbers that appear in our results; $N_{m}$ and $\pi(m)$.

Definition 5 . The closed path counting function $N_{m}$ is the number of closed paths $C$ of length $m$ in $X$ without backtracking or tails.

Definition 6. The prime counting function is

$$
\pi(n)=\#\{[C]=\text { primes in } X \text { with length of } C \text { equal to } n\} .
$$

Definition 7. The girth $X=$ length of the shortest closed path in $X$.

Note that the idea of a prime $[C]$ in a graph does not share too much with that of a prime number. In particular, one does not have unique factorization or even the commutative law. If $\left[P_{i}\right]$ are distinct primes, then $\left[P_{1}\right] \neq\left[P_{1}^{-1}\right]$, and $\left[P_{1} P_{2} P_{3}\right] \neq\left[P_{1} P_{3} P_{2}\right]=\left[P_{3} P_{2} P_{1}\right]$.

We will prove the following formulas involving the $N_{m}$ of Definition 5 in Section 3.

Kernel \#1) Define the Chebyshev polynomials of the first and second kind, respectively, by

$$
T_{n}(\cos \theta)=\cos (n \theta) \text { and } U_{n}(\cos \theta)=\frac{\sin ((n+1) \theta)}{\sin \theta} .
$$


The spherical function for $\mathbb{T}_{k}$ is

$$
h_{\lambda}(n)=q^{-n / 2}\left\{\frac{2}{q+1} T_{n}\left(\frac{\lambda}{2 \sqrt{q}}\right)+\frac{q-1}{q+1} U_{n}\left(\frac{\lambda}{2 \sqrt{q}}\right)\right\} .
$$

Let $g=$ girth of the $k=(q+1)$-regular connected finite graph $X, A=$ the adjacency operator on $X$, and $\operatorname{Spec}(A)=$ the spectrum of $A$. Note that the spectrum of $A$ is finite. Then for $r>2+g$,

$$
\begin{gathered}
k q^{r-1} \sum_{\lambda_{i} \in \operatorname{Spec}(A)} h_{\lambda_{i}}(r)= \\
N_{r}+(q-1)\left\{N_{r-2}+q N_{r-4}+\cdots+\left\{\begin{array}{ll}
N_{g} q^{\frac{r-g-2}{2}}, & \text { if } r-g \text { is even, } \\
N_{g+1} q^{\frac{r-g-1}{2}}, & \text { otherwise. }
\end{array}\right\}\right\}
\end{gathered}
$$

We can use formula (1.3) to show that if $X_{n}$ is a sequence of $(q+1)$-regular graphs such that for each $r$, we have $\frac{N_{r}}{\left|X_{n}\right|} \rightarrow 0$, then the spectrum of the adjacency matrix $A_{X_{n}}$ becomes equidistributed with respect to the measure given in Theorem 4. This measure approaches the semicircle distribution as the degree approaches infinity.

Kernel \#2) The trace formula for the heat kernel says:

$$
\sum_{\lambda \in \operatorname{Spec}(A)} e^{\frac{\lambda t}{k}}=\frac{2 q k|X|}{\pi} \int_{-1}^{1} e^{\frac{t u \sqrt{q}}{k}} \frac{\sqrt{1-u^{2}}}{k^{2}-4 q u^{2}} d u+\sum_{n \geq g} N_{n} q^{-n / 2} I_{n}\left(\frac{2 t \sqrt{q}}{k}\right),
$$

where $I_{n}(x)$ denotes the I-Bessel function whose generating function is

$$
e^{\frac{x}{2}\left(t+\frac{1}{t}\right)}=\sum_{n=-\infty}^{\infty} I_{n}(x) t^{n} .
$$

Kernel \#3) The trace formula for kernel \#3 implies that the Ihara zeta function is the reciprocal of a polynomial.

Definition 8. The Ihara zeta function for a finite connected graph $X$ is a function of the complex variable $u$ defined for $|u|$ sufficiently small by

$$
\zeta_{X}(u)=\prod_{[C]}\left(1-u^{\nu(C)}\right)^{-1}
$$

where $[C]$ runs over all primes of $X$.

Note that the product defining the Ihara zeta function is infinite unless the graph $X$ is a cycle graph. For more information on the Ihara zeta function, see Stark and Terras $[\mathbf{3 6}],[\mathbf{3 7}]$.

Theorem 4 in the last section of this paper implies that

$$
N_{m}=(r-1)\left\{1+(-1)^{m}\right\}+\sum_{\lambda_{i} \in \operatorname{Spec}(A)}\left\{\alpha_{\lambda_{i}}^{m}+\beta_{\lambda_{i}}^{m}\right\},
$$

where $1-\lambda_{i} u+q u^{2}=\left(1-\alpha_{\lambda_{i}} u\right)\left(1-\beta_{\lambda_{i}} u\right)$. This implies, for a $(q+1)$-regular graph $X$,

$$
\begin{aligned}
& N_{m} \sim q^{m}, \quad \text { as } m \rightarrow \infty, \quad \text { if } X \text { is non-bipartite; } \\
& N_{2 m} \sim 2 q^{2 m}, \quad \text { as } m \rightarrow \infty, \quad \text { if } X \text { is bipartite. }
\end{aligned}
$$


Note that $N_{2 m+1}=0$, if $X$ is bipartite. Here the graph $X$ is bipartite means that the set of vertices of $X$ is a disjoint union $A \cup B$, where the vertices in $A$ are adjacent only to vertices in $B$ and the vertices in $B$ are adjacent only to vertices in $A$. See some of the graph theory references mentioned above for the reflections of the bipartite nature of $X$ in the spectrum of $A$.

Kernel \#4) The sum of the horocycle transforms is divergent in general for this kernel.

Kernel \#5) Write $\lambda(w)=q^{w}+q^{1-w}$. Then Re $w>1$ implies

$$
\sum_{\lambda \in \operatorname{Spec}(A)} \frac{1}{\lambda-\lambda(w)}=\frac{k|X|}{2 \pi} \int_{-2 \sqrt{q}}^{2 \sqrt{q}} \frac{\sqrt{4 q-\lambda^{2}}}{k^{2}-\lambda^{2}} \frac{1}{\lambda-\lambda(w)} d \lambda-\sum_{n \geq g} N_{n} \frac{q^{-w|n|}}{q^{w}-q^{1-w}}
$$

Kernel \#6) For any positive integer $n$, we have, writing $\lambda_{s}=q^{s}+q^{1-s}$,

$$
\sum_{\lambda_{s} \in \operatorname{Spec}(A)}\left(q^{n s}+q^{n(1-s)}\right)=N_{n}+ \begin{cases}-(q-1)|X|, & \text { if } n \text { is even } \\ 0, & \text { if } n \text { is odd. }\end{cases}
$$

This gives a quicker proof of the asymptotic formula (1.7) than that from kernel \#3. It is not hard to deduce the graph prime number theorem from this, assuming the basic facts about the spectrum of $A$, given in the graph theory references mentioned above.

Theorem 1. (Graph Prime Number Theorem) Suppose $X$ is a $(q+1)$ regular connected finite graph. Recall Definition 6 of $\pi(m)$. Then we have the following results.

If $X$ is non-bipartite, $\pi_{X}(m) \sim \frac{q^{m}}{m}$ as $m \rightarrow \infty$.

If $X$ is bipartite, $\pi_{X}(m)=0$ if $m$ is odd and $\pi_{X}(2 m) \sim \frac{q^{2 m}}{m}$ as $m \rightarrow \infty$.

Proof. First note that

$$
N_{m}=\sum_{d \mid m} d \pi(d) .
$$

Thus Möbius inversion says that if the Möbius function is

$$
\mu(n)= \begin{cases}1, & n=1 \\ (-1)^{k}, & n=p_{1} \ldots p_{k}, \text { distinct primes } \\ 0, & n \text { is divisible by the square of a prime, }\end{cases}
$$

then $\pi(m)=\frac{1}{m} \sum_{d \mid m} N_{d} \mu\left(\frac{m}{d}\right)$. The prime number theorem then follows from formula (1.7).

\section{Selberg Trace Formula for the $k$-regular Tree}

The Selberg Trace Formula (STF) has been around for at least 50 years. Originally, Selberg [34] used it to study discrete groups $\Gamma$ of Möbius transformations of the Poincaré upper half plane $\mathbb{H}$. It can be used to prove

(1) properties of the Selberg Zeta function for the Riemann surface $\Gamma \backslash \mathbb{H}$,

(2) the Weyl law for asymptotics of the spectrum of the Laplacian, $\Delta$, on $\Gamma \backslash \mathbb{H}$ (for $\Gamma$ co-compact or arithmetic), and

(3) the Prime Number Theorem for lengths of prime geodesics in $\Gamma \backslash \mathbb{H}$. 
Here we replace $\mathbb{H}$ by the $k$-regular tree, $\mathbb{T}_{k}$ for $k=q+1$, and $\Gamma$ by the fundamental group of a finite graph $X$. See Figure 1 for part of the 4-regular tree. We will normally assume $k>2$ as the $k=2$ case is trivial and different in many ways. However, it is a useful exercise to do this case. We will sketch the background of the trace formula (due to Ahumada [1]). References for more information are Brooks [7], Cartier [8], [9], Figà-Talamanca and Nebbia [16], Hurt $[\mathbf{2 2}]$, Nagoshi $[\mathbf{3 0}]$ and $[\mathbf{3 1}]$, Quenell $[\mathbf{3 3}]$, Sunada $[\mathbf{3 8}]$, Terras $[\mathbf{4 1}]$ and $[\mathbf{4 2}]$, Terras and Wallace [43], Venkov and Nikitin [46].

\subsection{Adjacency Operator, Geodesics, and Horocycles.}

Definition 9. The Hilbert space for $\mathbb{T}_{k}$ is

$$
\begin{aligned}
L^{2}\left(\mathbb{T}_{k}\right) & =\left\{f:\left.\mathbb{T}_{k} \rightarrow \mathbb{C}\left|\sum_{x \in \mathbb{T}_{k}}\right| f(x)\right|^{2}<\infty\right\}, \text { with inner product } \\
(f, g) & =\sum_{x \in \mathbb{T}_{k}} f(x) \overline{g(x)},\|f\|=(f, f)^{\frac{1}{2}} .
\end{aligned}
$$

Definition 10. The adjacency operator, $\widetilde{A}$, on $\mathbb{T}_{k}$ is defined for $f \in L^{2}\left(\mathbb{T}_{k}\right)$ by

$$
(\widetilde{A} f)(x)=\sum_{\substack{y \in \mathbb{T}_{k} \\ y \text { adjacent to } x}} f(y) .
$$

Proposition 1. Given $f, g \in L^{2}\left(\mathbb{T}_{k}\right)$ and $\widetilde{A}$, the adjacency operator on $\mathbb{T}_{k}$, we have the following facts.

(1) $(\widetilde{A} f, g)=(f, \widetilde{A} g)$,

(2) $\widetilde{A}-k I=\widetilde{\Delta} \Longrightarrow(\widetilde{\Delta} f, f) \leq 0$, and

(3) $|(\widetilde{A} f, f)| \leq 2 \sqrt{q}(f, f), q=k-1$.

Proof. See Sunada $[\mathbf{3 8}]$.

It follows that the spectrum of $\widetilde{A}$ on $\mathbb{T}_{k}$ is contained in the interval $[-2 \sqrt{q}, 2 \sqrt{q}]$. In fact, the spectrum of $\widetilde{A}$ on $\mathbb{T}_{k}$ equals the interval $[-2 \sqrt{q}, 2 \sqrt{q}]$ and is continuous in the sense of spectral theory (see Terras $[\mathbf{4 0}]$ ). The spectral theorem for selfadjoint operators writes $f \in L^{2}\left(\mathbb{T}_{k}\right)$ as an integral over the spectrum of $\widetilde{A}$. We will say more about this later. First, we need some basics about the geometry of $\mathbb{T}_{k}$.

Definition 11. A geodesic $\left\{x_{n}\right\}_{n \in \mathbb{Z}}$ is a doubly infinite path in $\mathbb{T}_{k}$.

Definition 12. A chain, $C=\left\{x_{n}\right\}_{n>0}$ in $\mathbb{T}_{k}$ is a semi-infinite path or ray.

So we can view a geodesic as the union of 2 chains, both starting at the same vertex $x_{0}$.

Definition 13. The boundary, $\Omega$, of $\mathbb{T}_{k}$ consists of equivalence classes of chains, where $C \sim D \Leftrightarrow C$ and $D$ coincide except for a finite number of vertices.

Definition 14. A chain connecting $x \in \mathbb{T}_{k}$ to $\infty$ along $\omega \in \Omega$ is $[x, \omega]$.

Take $C \in \omega$ and let $y$ be a point of $C$ with a minimum distance to $x$. Make $[x, \omega]$ by joining $x$ to $y$ and then continuing along $C$. See Figure 2 . 


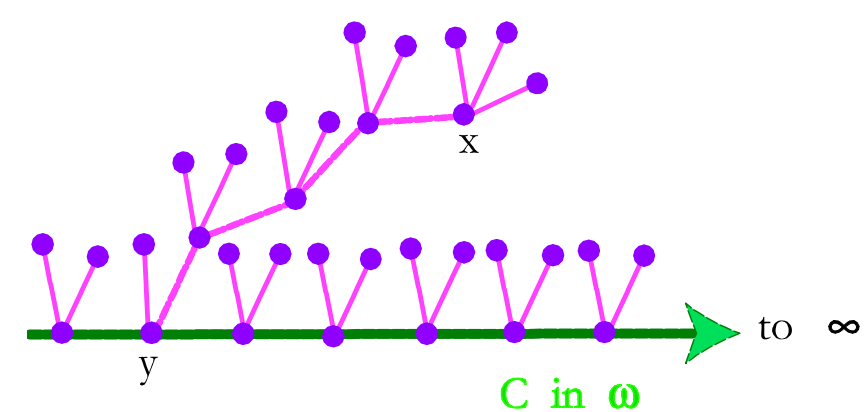

Figure 2. A chain connecting $x \in T_{k}$ to $\infty$ along $\omega \in \Omega$ is $[x, \omega]$.

Definition 15. For $x, y \in \mathbb{T}_{k}, x \sim y \Longleftrightarrow[x, \omega] \cap[y, \omega]=[z, \omega]$ and $d(x, z)=$ $d(y, z)$. These equivalence classes are called $\omega$-horocycles.

If we fix a boundary element $\omega$ of $\mathbb{T}_{k}$ with chain $C \in \omega$ and geodesic $C \cup C^{\prime}$ then we can label the horocycles with numbers $n \in \mathbb{Z}$ as in Figure 3.

Definition 16. Set $\mathfrak{h}_{n}=\{$ points labelled $n\}=n^{\text {th }}$ horocycle.

The horocycle $\mathfrak{h}_{n}$ is infinite. In fact, letting $o$ denote some fixed point of $\mathbb{T}_{k}$, which we call the origin, we have the formula:

$$
\begin{aligned}
S(n, d)= & \#\left\{x \in \mathfrak{h}_{n} \mid d(x, o)=d\right\} \\
= & \begin{cases}q^{n}(q-1) q^{\frac{d-n}{2}}-1, & \text { if } 2 \mid(d-n), d>n \geq 0, \\
(q-1) q^{\frac{d-|n|}{2}-1}, & \text { if } 2|(d-|n|), n<0, d>| n \mid, \\
q^{n}, & \text { if } d=n>0, \\
1, & \text { if } d=n=0, \\
0, & \text { if } d<|n| \text { or } 2 \nmid(d-|n|) .\end{cases}
\end{aligned}
$$

According to Figà-Talamanca and Nebbia [16], page 25, horocycles can be viewed as "generations with respect to a common mythical ancestor $\omega$." See Figure 3.

The Selberg trace formula involves two sorts of transforms. One is the horocycle transform.

Definition 17. The horocycle transform of $f: \mathbb{T}_{k} \rightarrow \mathbb{C}$ is $F(\mathfrak{h})=\sum_{x \in \mathfrak{h}} f(x)$ for any horocycle $\mathfrak{h}$ of $\mathbb{T}_{k}$.

Assuming $f$ is finitely supported and invariant under rotation about the origin, $o$, we can write $f(x)=f(d(x, o))$ and then we have, for $k=q+1$,

$$
F\left(\mathfrak{h}_{n}\right)=c_{n} H f(n),
$$




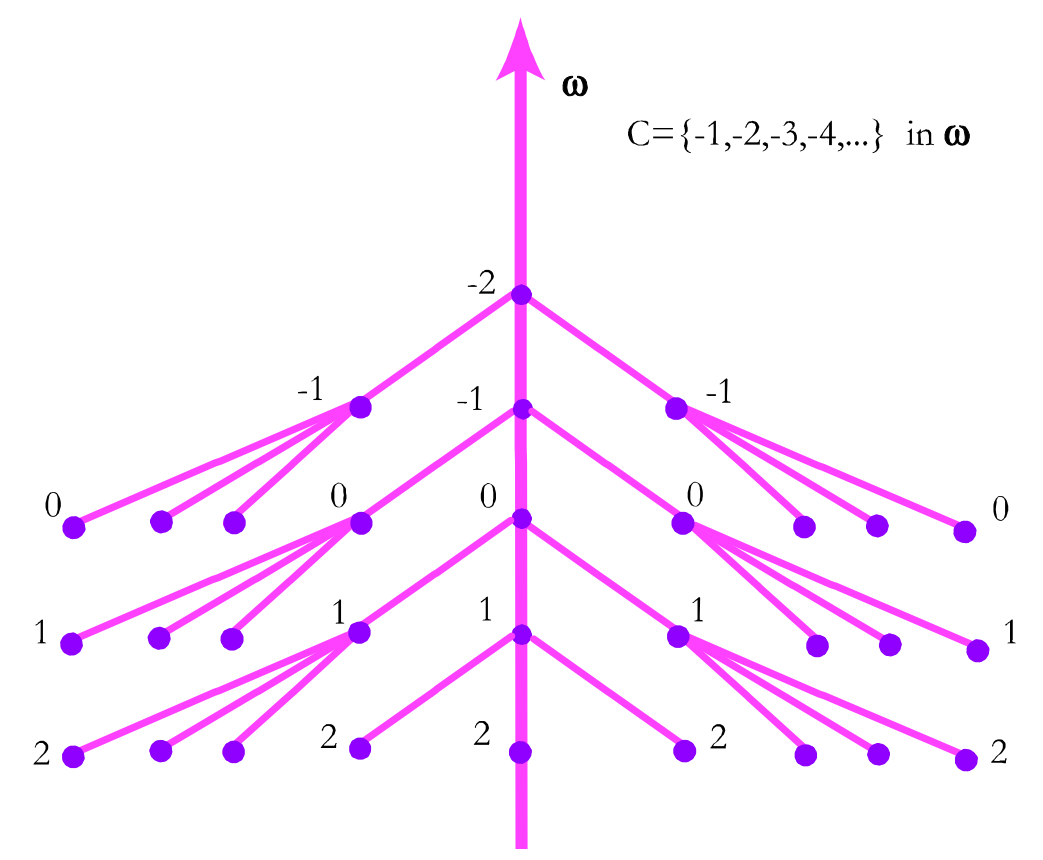

FiguRE 3. Horocycles are points labelled with the same integer.

where $c_{n}=\left\{\begin{array}{cc}q^{n}, & n>0, \\ 1, & n \leq 0,\end{array}\right.$ and

$$
H f(|n|)=f(|n|)+(q-1) \sum_{j=1}^{\infty} q^{j-1} f(|n|+2 j) .
$$

This transform is invertible.

$$
f(|n|)=H f(|n|)-(q-1) \sum_{j \geq 1}(H f)(|n|+2 j)
$$

2.2. Spherical Functions and Transforms. The second sort of transform occurring in the Selberg Trace Formula on $\mathbb{T}_{k}$ is the spherical transform associated to spherical functions on $\mathbb{T}_{k}$.

Note that the graph isomorphism group $\mathcal{G}$ of $\mathbb{T}_{k}$ has 3 types of elements:

(1) Rotations (which fix a vertex);

(2) Inversions (which fix an edge and exchange the endpoints);

(3) Hyperbolic elements ( $\rho$ fixes a geodesic $\left\{x_{n}\right\}_{n \in \mathbb{Z}}, \rho\left(x_{n}\right)=x_{n+s}$, where we define $s=\nu(\rho))$.

Fix $o$, the origin of $\mathbb{T}_{k}$. If we let $\mathcal{K}=$ group of rotations of $\mathbb{T}_{k}$ fixing $o$, and since $\mathcal{G}=\operatorname{Aut}\left(\mathbb{T}_{k}\right)$, we see that $(\mathcal{G}, \mathcal{K})$ is a Gelfand pair. That is, $L^{1}(\mathcal{K} \backslash \mathcal{G} / \mathcal{K})$ is a commutative algebra under convolution on the group $\mathcal{G}$. Here we can identify $\mathbb{T}_{k}$ with $\mathcal{G} / \mathcal{K}$. See Terras [41] for more information on Gelfand pairs.

Definition 18. A function $h: \mathbb{T}_{k} \rightarrow \mathbb{C}$ is a spherical function $\Leftrightarrow h$ has the following 3 properties:

(1) $h(x)=h(\kappa x)$, for all rotations $\kappa$ about $o$, 
(2) $\widetilde{A} h=\lambda h$; i.e., $h$ is an eigenfunction of the adjacency operator $\widetilde{A}$ on $\mathbb{T}_{k}$, and

(3) $h(o)=1$.

It is not hard to use the definitions to obtain explicit formulas for $h$. See Brooks [7], Cartier [8], Figà-Talamanca and Nebbia [16], and Terras and Wallace [43]. We shall just state these results. In particular, letting $d=d(x, o)$ for $x \in \mathbb{T}_{k}$ and $s \in \mathbb{C}$, for $\lambda=q^{s}+q^{1-s}$, the spherical function, $h_{s}$, corresponding to an eigenvalue, $\lambda$, is unique and can be written as

$$
h_{s}(d)=c(s) q^{-s d}+c(1-s) q^{-(1-s) d},
$$

where $c(s)$ is the Harish-Chandra c-function

$$
c(s)=\frac{1}{q+1} \frac{q^{s-1}-q^{1-s}}{q^{s-1}-q^{-s}}
$$

assuming $q^{2 s-1} \neq 1$. An alternate form of this equation is

$$
h_{s}(x)=h_{s}(d)=\frac{1}{q^{\frac{d}{2}}} \frac{1}{q+1} \frac{1}{z^{d}}\left(q \frac{z^{2+2 d}-1}{z^{2}-1}-\frac{z^{2 d}-z^{2}}{z^{2}-1}\right),
$$

where $z=q^{s-\frac{1}{2}}$ and we assume $z^{2} \neq 1$. If $z^{2}=1$, take limits. Sometimes we will write $h_{\lambda}(d)$ rather than $h_{s}(d)$.

It follows that

$$
\begin{aligned}
h_{\lambda}(n) & =q^{-\frac{n}{2}}\left(\frac{2}{q+1} T_{n}\left(\frac{\lambda}{2 \sqrt{q}}\right)+\frac{q-1}{q+1} U_{n}\left(\frac{\lambda}{2 \sqrt{q}}\right)\right) \\
& =q^{-\frac{n}{2}} G_{n}\left(\frac{\lambda}{2 \sqrt{q}}\right)
\end{aligned}
$$

where $T_{n}$ and $U_{n}$ are the Chebyshev polynomials of the first and second kind (see formula (1.1)), and $G_{n}$ is the Geronimus polynomial. See Solé [35] and Li and Solé $[\mathbf{2 6}]$.

The recursion for $h_{\lambda}(n)$ is

$$
q h_{\lambda}(n+1)=\lambda h_{\lambda}(n)-h_{\lambda}(n-1) .
$$

Definition 19. The spherical transform of a rotation-invariant function $f: \mathbb{T}_{k} \rightarrow \mathbb{C}$ is

$$
\widehat{f}(s)=\sum_{x \in \mathbb{T}_{k}} f(x) \overline{h_{s}(x)}=\left(f, h_{s}\right)_{\mathbb{T}_{k}} .
$$

We will also write $\widehat{f}(\lambda)=\widehat{f}(s)$, if $\lambda=q^{s}+q^{1-s}$.

Let us consider the properties of the spherical transform. The Plancherel theorem (or inversion formula) says for $s=\frac{1}{2}+i t, x \in \mathbb{T}_{k}$

$$
f(x)=\int_{0}^{\frac{\pi}{\log (q)}} \widehat{f}\left(\frac{1}{2}+i t\right) h_{s}(x) \frac{q \log (q)}{2 \pi(q+1)\left|c\left(\frac{1}{2}+i t\right)\right|^{2}} d t .
$$

Here, $c(s)$ is as defined in formula (2.3). Note: $f \rightarrow \widehat{f}$ provides an isometry between the $L^{2}$ spaces.

The Plancherel Theorem makes explicit the spectral resolution of $\widetilde{A}$ on $\mathbb{T}_{k}$. See Figà-Talamanca and Nebbia [16], page 61, for a computation of the spectral 
measure from the Kodaira, Titchmarsh, Carleman, Stieltjes, Stone, etc. formula involving the resolvent kernel or Green's function, which is Kernel \#5. See Terras [40], p. 111, for a brief discussion of the Kodaira-Titchmarsh formula.

Definition 20. Assume $f, g$ are rotation-invariant with finite support. Then the convolution of $f$ and $g$ is defined by

$$
(f * g)(d(x, z))=\sum_{y \in \mathbb{T}_{k}} f(d(x, y)) g(d(y, z)) .
$$

Definition 21. Define the Schwartz space $\mathcal{S}$ as the set of all functions $f: \mathbb{T}_{k} \rightarrow \mathbb{C}$ such that $f$ is rotation-invariant with finite support.

Proposition 2. (Properties of Convolution). For $f, g, h \in \mathcal{S}$, we have the following facts.

(1) $f * g=g * f$.

(2) For $\alpha, \beta \in \mathbb{C},(\alpha f+\beta g) * h=\alpha(f * h)+\beta(g * h)$.

(3) $(f * g) * h=f *(g * h)$.

(4) $\widehat{f * g}(\lambda)=\widehat{f}(\lambda) \cdot \widehat{g}(\lambda)$.

Proof. We leave the proofs of (1)-(3) to the reader. To prove (4), it suffices to assume $f$ and $g$ come from a basis of $\mathcal{S}$. Thus, it suffices to let $f=f_{m}$, and $g=f_{n}$, where $f_{n}$ is kernel $\# 1$. First note that if $z=o$,

$$
\begin{aligned}
\left(h * f_{n}\right)(d(x, o)) & =\sum_{y} h(d(x, y)) f_{n}(d(y, o)) \\
& =\sum_{y \text { s.t. } d(y, o)=n} h(d(x, y))=\left(\Theta_{n} h\right)(d(x, o)),
\end{aligned}
$$

where $\Theta_{n}$ is the $n^{\text {th }}$ Hecke operator. See Cartier [8], [9].

The Hecke operators form a commutative algebra generated by $\Theta_{0}=$ Identity, $\Theta_{1}=\widetilde{A}=$ adjacency operator. We can view this algebra as $\mathcal{S}$ under $*$. The spherical functions $h_{\lambda}$ are eigenfunctions of this algebra since $\Theta_{1} h_{\lambda}=\lambda h_{\lambda}$ and we find

$$
\begin{aligned}
\Theta_{2} h_{\lambda} & =\left(\Theta_{1}^{2}-k I\right) h_{\lambda}=\left(\lambda^{2}-k\right) h_{\lambda}, \text { and } \\
\Theta_{n+1} h_{\lambda} & =\left(\Theta_{1} \Theta_{n}-q \Theta_{n-1}\right) h_{\lambda} .
\end{aligned}
$$

So for $n \geq 1$, by induction on $n$, we have (recalling (2.6))

$$
\left(f_{n} * h_{\lambda}\right)=\Theta_{n} h_{\lambda}=h_{\lambda}(n)(q+1) q^{n-1} h_{\lambda}=\widehat{f_{n}}(\bar{\lambda}) h_{\lambda} .
$$

Thus if $n, m \geq 0$ we have

$$
\begin{aligned}
\widehat{f_{n} * f_{m}}(\bar{\lambda}) h_{\lambda} & =\left(\left(f_{n} * f_{m}\right) * h_{\lambda}\right)=f_{n} *\left(f_{m} * h_{\lambda}\right) \\
& =f_{n} *\left(\widehat{f_{m}}(\bar{\lambda}) h_{\lambda}\right)=\widehat{f_{m}}(\bar{\lambda})\left(f_{n} * h_{\lambda}\right)=\widehat{f_{m}}(\bar{\lambda}) \widehat{f_{n}}(\bar{\lambda}) h_{\lambda}
\end{aligned}
$$

Property (4) follows.

Lemмa 1. (Relation between Spherical and Horocycle Transforms) Suppose $f \in \mathcal{S}$ from Definition 21. Then, if $z=q^{s-\frac{1}{2}}, \lambda=q^{s}+q^{1-s}$,

$$
\widehat{f}(\bar{s})=\left(f, \overline{h_{s}}\right)_{\mathbb{T}_{k}}=\sum_{n \in \mathbb{Z}}(H f)(n) q^{\frac{|n|}{2}} z^{n} .
$$

Proof. See Terras and Wallace [43], p. 511 for some hints. 
Corollary 1. Let $f$ and $z$ be as in the preceding Lemma. Assume $|z|=1$ and $\widehat{f}(\bar{s})=\widehat{f}(\bar{z})$ is a Fourier series of horocycle transforms which implies

$$
(H f)(n) q^{\frac{|n|}{2}}=\frac{1}{2 \pi i} \int_{|z|=1} \widehat{f}(\bar{z}) z^{-n} \frac{d z}{z} .
$$

Lemma 2. (Behavior of the Spherical function). If $\lambda=q^{s}+q^{1-s}$, $\frac{1}{2} \leq \operatorname{Re} s<1$, then $\lim _{d \rightarrow \infty} h_{s}(d)=0$.

Proof. Recall that

$$
\begin{aligned}
h_{s}(d) & =c(s) q^{-s d}+c(1-s) q^{-(1-s) d} \\
& =\frac{1}{q^{\frac{d}{2}}} \frac{1}{q+1} \frac{1}{z^{d}}\left(q \frac{z^{2+2 d}-1}{z^{2}-1}-\frac{z^{2 d}-z^{2}}{z^{2}-1}\right),
\end{aligned}
$$

where $z=q^{s-\frac{1}{2}}$. Since $\left|q^{-s d}\right|=q^{-(\operatorname{Re} s) d}$ and $\left|q^{-(1-s) d}\right|=q^{-\operatorname{Re}(1-s) d}$, when $z^{2} \neq 1$, we have $h_{s}(d) \rightarrow 0$ as $d \rightarrow \infty$. If $z^{2}=1$, then $\left|h_{s}(d)\right|=\frac{1}{q^{\frac{d}{2}}}\left|1+\frac{q-1}{q+1} d\right| \rightarrow 0$ as $d \rightarrow \infty$. Note that we are assuming that $q>1$.

Lemma 3. Let $\psi: \mathbb{T}_{k} \rightarrow \mathbb{C}$ be any eigenfunction of the adjacency operator $\widetilde{A}$; i.e., $\widetilde{A} \psi=\lambda \psi$. Define $f_{r}$ to be kernel \#1. Then

$$
\sum_{y \in \mathbb{T}_{k}} f_{r}(x, y) \psi(y)= \begin{cases}k(k-1)^{r-1} h_{s_{\lambda}}(r) \psi(x), & r>0 \\ \psi(x), & r=0 .\end{cases}
$$

Here $h_{s_{\lambda}}(x)=h_{s_{\lambda}}(d(x, o))$ is the spherical function corresponding to the eigenvalue $\lambda=q^{s_{\lambda}}+q^{1-s_{\lambda}}$.

Proof. Use formulas (2.8) and (2.9).

Corollary 2. (Selberg's Lemma) If $f \in \mathcal{S}, \psi$ is an eigenfunction of $\widetilde{A}$ on $\mathbb{T}_{k}$ with $\widetilde{A} \psi=\lambda \psi, \lambda=q^{s_{\lambda}}+q^{1-s_{\lambda}}, o=$ origin of $\mathbb{T}_{k}$, then

$$
(f, \bar{\psi})_{\mathbb{T}_{k}}=\psi(o)\left(f, \bar{h}_{s_{\lambda}}\right)_{\mathbb{T}_{k}}
$$

where $(f, \bar{g})_{\mathbb{T}_{k}}=\sum_{x \in \mathbb{T}_{k}} f(x) g(x)$.

Proof. Use Lemma 3 and the fact that $f$ is a finite linear combination of the $f_{n}$

2.3. Pre-Trace Formula. Next we consider the pre-trace formula associated to a finite, connected, k-regular graph $X$. Note that $\mathbb{T}_{k}$ is the universal covering graph of $X$ with $\pi: \mathbb{T}_{k} \rightarrow X$ the covering map. One can identify $X$ with $\Gamma \backslash \mathbb{T}_{k}$, where $\Gamma=$ group of deck transformations of the covering (i.e., the Galois group of the covering in the sense of Stark and Terras $[\mathbf{3 7}]$ ). Here $\Gamma$ can be identified as the fundamental group of $X$ (a group generated by paths associated to edges left out of a spanning tree of $X$ ).

In this case $\rho \in \Gamma, \rho \neq$ identity implies $\rho$ is a hyperbolic element of the automorphism group of $\mathbb{T}_{k}$. Note also that $\Gamma$ is a free group on $r$ generators, $r=|E|-|V|+1$.

Definition 22. A hyperbolic element $\rho \in \Gamma$ is primitive if and only if it generates $\Gamma_{\rho}=\{\gamma \in \Gamma \mid \gamma \rho=\rho \gamma\}$, the centralizer of $\rho$. 
The group $\Gamma_{\rho}$ is cyclic since $\Gamma$ is free.

DEFinition 23. The set of $\{\rho\}$ such that $\rho$ is a primitive hyperbolic element of $\Gamma$ is called $\wp_{\Gamma}$.

We will now look at the connection between $\{\rho\} \in \wp_{\Gamma}$ and prime paths $[C]$ in $X=\Gamma \backslash \mathbb{T}_{k}$.

Given $\{\rho\} \in \wp_{\Gamma}$, we note that $\rho$ fixes a geodesic $R$ in $\mathbb{T}_{k}$. Consider for example $\rho$ in Figure 4 which shifts by $\nu(\rho)=3$ along the geodesic at the top of the figure. Then the projection $\pi$ from $\mathbb{T}_{4}$ to $X$ maps the geodesic $R$ fixed by $\rho$ onto the prime path $[C]$ through vertices $1,2,3$ and then back to 1 in $K_{5}$.

It is easily seen that for $\gamma \in \Gamma, \gamma R$ is the geodesic fixed by $\gamma \rho \gamma^{-1}$. Both $R$ and $\gamma R$ project under $\pi$ to the same path $[C]$ in $X$. Conversely, given $[C]$ in $X$, we can identify $\rho \in \Gamma$ corresponding to $[C]$ with its Frobenius automorphism, defined in $[\mathbf{3 7}]$, which is a conjugacy class in $\Gamma$.

\section{$\mathrm{T}_{4}$}
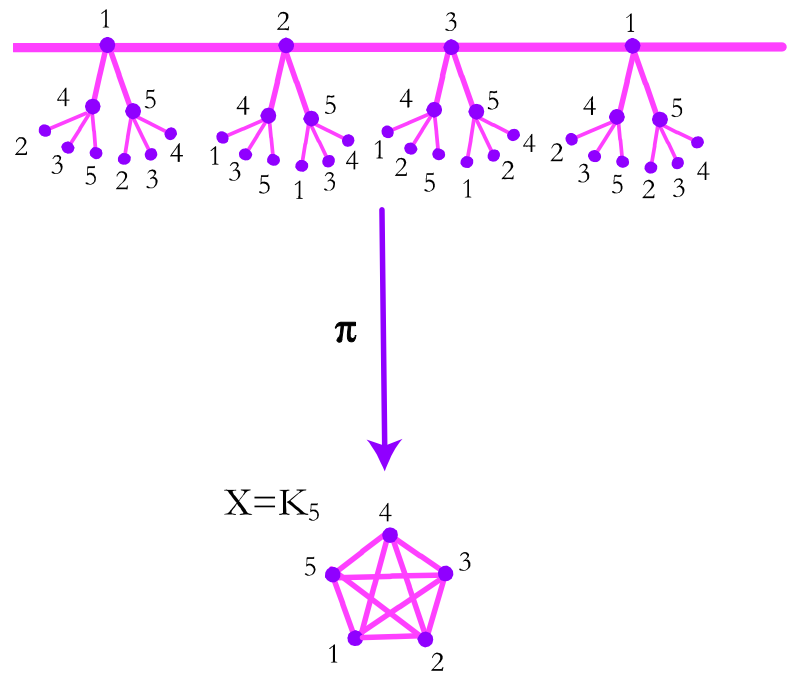

FigurE 4. Each point of $\mathbb{T}_{4}$ is labelled with its image vertex in $K_{5}$ under the projection map $\pi$.

Definition 24. We lift functions $\phi: X \rightarrow \mathbb{C}$ to $\psi: \mathbb{T}_{k} \rightarrow \mathbb{C}$, via $\psi(x)=$ $\phi(\pi(x))$ where $\pi: \mathbb{T}_{k} \longrightarrow X$ is the covering map and $\widetilde{A} \psi=\widetilde{A} \phi$.

So, if we let $\left\{\phi_{i}\right\}_{i=1, \ldots, n=|X|}$ be a complete orthonormal set of eigenfunctions of $A$ on $L^{2}(X)$ with inner product

$$
(f, g)=\sum_{x \in X} f(x) \overline{g(x)},
$$

we can plug $\phi_{i}$ into Selberg's Lemma. Compare the following lemma with Mercer's theorem in Courant and Hilbert [12], p. 138. 
Lemma 4. (Mercer's Theorem on $\mathbb{T}_{k}$ ) Let $\left\{\phi_{i}\right\}_{i=1, \ldots, n}$ be a complete orthonormal set of eigenfunctions of $A$ on $X$. Suppose $f \in \mathcal{S}$ as in Definition 21. Write $f(y)=f(d(y, x))$. Then, defining for $x, y \in \mathbb{T}_{k}$,

$$
K_{f}(x, y)=\sum_{\gamma \in \Gamma} f(d(x, \gamma y)),
$$

we have

$$
K_{f}(x, y)=\sum_{i=1}^{|X|=n}\left(f, h_{s_{\lambda_{i}}}\right)_{\mathbb{T}_{k}} \overline{\psi_{i}(x)} \psi_{i}(y) .
$$

Here $\psi_{i}$ is the lift of $\phi_{i}$ as in Definition 24. Note that $K_{f}\left(\gamma_{1} x, \gamma_{2} y\right)=K_{f}(x, y)$ for all $\gamma_{1}, \gamma_{2} \in \Gamma \Rightarrow K_{f}$ is a function on $X \times X$.

Proof. Note that $K_{f}(x, y)=\sum_{i, j} c_{i j} \overline{\psi_{i}(x)} \psi_{j}(y)$ where

$$
\begin{aligned}
c_{i j} & =\sum_{x, y \in X} K_{f}(x, y) \psi_{i}(x) \overline{\psi_{j}(y)} \\
& =\sum_{x \in X}\left(\sum_{y \in X} K_{f}(x, y) \overline{\psi_{j}(y)}\right) \psi_{i}(x) .
\end{aligned}
$$

Then we have

$$
\begin{aligned}
\sum_{y \in X=\Gamma \backslash \mathbb{T}_{k}} K_{f}(x, y) \overline{\psi_{j}(y)} & =\sum_{y \in X=\Gamma \backslash \mathbb{T}_{k}} \sum_{\gamma \in \Gamma} f(d(x, \gamma y)) \overline{\psi_{j}(\gamma y)} \\
& =\sum_{y \in \mathbb{T}_{k}} f(d(x, y)) \overline{\psi_{j}(y)} \\
& =\left(f, \psi_{j}\right)_{\mathbb{T}_{k}}=\overline{\psi_{j}(o)}\left(f, h_{s_{\lambda}}\right)_{\mathbb{T}_{k}},
\end{aligned}
$$

by Selberg's Lemma, since we may choose the origin, $o$, of $\mathbb{T}_{k}$ to be the arbitrary point $x$.

It is important to notice that $\psi(\gamma y)=\phi(\pi(\gamma y))=\phi(\pi(y))=\psi(y)$. Therefore,

$$
\begin{aligned}
c_{i j} & =\sum_{x \in X} \overline{\psi_{j}(x)}\left(f, h_{s_{\lambda_{j}}}\right)_{\mathbb{T}_{k}} \psi_{i}(x) \\
& = \begin{cases}0, & i \neq j \\
\left(f, h_{s_{\lambda_{j}}}\right)_{\mathbb{T}_{k}}, & i=j\end{cases}
\end{aligned}
$$

since the $\psi$ are orthonormal.

Let $\phi: X \rightarrow \mathbb{C}$ and $\psi: \mathbb{T}_{k} \rightarrow \mathbb{C}$ be the lift of $\phi$. Let $f: \mathbb{T}_{k} \rightarrow \mathbb{C}$ be a rotation-invariant, finitely supported function. Then

$$
\begin{aligned}
\left(L_{f} \psi\right)(x) & =\sum_{y \in \mathbb{T}_{k}} f(d(x, y)) \psi(y) \\
& =\sum_{y \in X=\Gamma \backslash \mathbb{T}_{k}} \sum_{\gamma \in \Gamma} f(d(x, \gamma y)) \psi(y) .
\end{aligned}
$$


Thus, using Mercer's theorem,

$$
\begin{aligned}
\left.\operatorname{Tr}\left(L_{f}\right)\right|_{L^{2}\left(X=\Gamma \backslash \mathbb{T}_{k}\right)} & =\sum_{x \in X} K_{f}(x, x) \\
& =\sum_{x \in X} \sum_{i=1}^{|X|}\left(f, h_{s_{\lambda_{i}}}\right)_{\mathbb{T}_{k}} \overline{\psi_{i}(x)} \psi_{i}(x)=\sum_{i=1}^{|X|} \widehat{f}\left(\lambda_{i}\right),
\end{aligned}
$$

where $\lambda_{i} \in \operatorname{Spec}(A) \subset \mathbb{R}$. This gives us the following theorem.

Theorem 2. (Pre-Trace Formula). For functions $f$ in $\mathcal{S}$, we have

$$
\left.\operatorname{Tr}\left(L_{f}\right)\right|_{L^{2}\left(X=\Gamma \backslash \mathbb{T}_{k}\right)}=\sum_{\lambda_{i} \in \operatorname{Spec}(A)} \widehat{f}\left(\lambda_{i}\right)=\sum_{x \in X} \sum_{\gamma \in \Gamma} f(d(x, \gamma x)) .
$$

The left hand side is a sum over the spectrum of the adjacency operator $A$ on $X$ and $\widehat{f}(\lambda)$ is the spherical transform of $f$ from Definition 19 .

2.4. The Trace Formula. Now to get the trace formula itself, we must rewrite the right hand side of Theorem 2 as a sum over conjugacy classes $\{\gamma\}$ in $\Gamma$.

Definition 25. The orbital sum $I_{\gamma}(f)=\sum_{\tau \in \Gamma_{\gamma} \backslash \mathbb{T}_{k}} f(d(x, \tau x))$.

So with this definition, the right hand side of Theorem 2 is

$$
\begin{aligned}
\sum_{x \in X} \sum_{\gamma \in \Gamma} f(d(x, \gamma x)) & =\sum_{x \in X=\Gamma \backslash \mathbb{T}_{k}} \sum_{\gamma \in \Gamma} f(d(x, \gamma x)) \\
& =\sum_{x \in X} \sum_{\{\gamma\}} \sum_{\tau \in\{\gamma\}} f(d(x, \tau x)) \\
& =\sum_{x \in \Gamma \backslash \mathbb{T}_{k}} \sum_{\{\gamma\}} \sum_{\tau \in \Gamma_{\gamma} \backslash \Gamma} f(d(x, \tau x)) \\
& =\sum_{\{\gamma\}}\left(\sum_{\tau \in \Gamma_{\gamma} \backslash \mathbb{T}_{k}} f(d(x, \tau x))\right)=\sum_{\{\gamma\}} I_{\gamma}(f),
\end{aligned}
$$

where $\{\gamma\}$ runs over all conjugacy classes of $\Gamma$ and $I_{\gamma}(f)$ is an orbital sum.

Lemma 5. For $\gamma \in \Gamma$,

(1) if $\{\gamma\}=\{$ Identity $\}$, then

$$
I_{\{I\}}(f)=\sum_{\Gamma \backslash \mathbb{T}_{k}=X} f(o)=f(o)|X| .
$$

Here $\Gamma_{I}=\Gamma$.

(2) if $\{\gamma\}$ is a hyperbolic element, then $\gamma=\rho^{r}$, where $\{\rho\} \in \wp_{\Gamma}$ and

$$
\begin{aligned}
I_{\left\{\rho^{r}\right\}}(f) & =\sum_{\langle\rho\rangle \backslash \mathbb{T}_{k}} f\left(d\left(x, \rho^{r} x\right)\right) \\
& =\nu(\rho) H f(r \nu(\rho)) .
\end{aligned}
$$

Proof. (1) Clear 


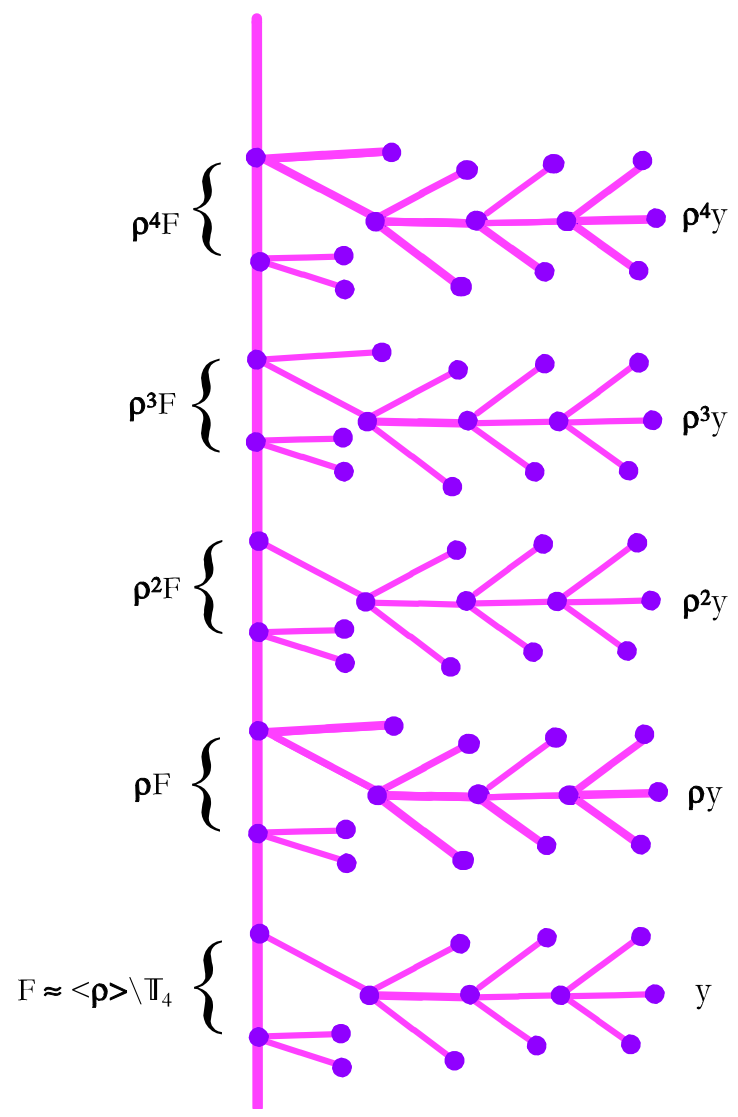

FiguRE 5. The geodesic fixed by a hyperbolic element $\rho$ and fundamental domains for $\left\langle\rho^{j}\right\rangle \backslash \mathbb{T}_{k}, j=1,2,3,4$.

(2) The quotient $\langle\rho\rangle \backslash \mathbb{T}_{k}$ is found by looking at the geodesic fixed by $\rho$, where $\Gamma_{\rho}=\langle\rho\rangle$. So, the number of elements in

$$
\left\{y \in \Gamma_{\rho} \backslash \mathbb{T}_{k} \mid d\left(y, \rho^{r} y\right)=r \nu(\rho)+2 j\right\}
$$

is equal to $\nu(\rho)(q-1) q^{j-1}$. See Figure 5. Therefore,

$$
\begin{aligned}
I_{\left\{\rho^{r}\right\}}(f) & =\sum_{y \in \Gamma_{\rho} \backslash \mathbb{T}_{k}} f(d(y, \rho y)) \\
& =\nu(\rho) f(r \nu(\rho))+\nu(\rho)(q-1) \sum_{j \geq 1} q^{j-1} f(r \nu(\rho)+2 j) \\
& =\nu(\rho) H f(r \nu(\rho)) .
\end{aligned}
$$

Collecting what we have proved leads finally to the trace formula. 
Theorem 3. (Selberg Trace Formula for k-regular, connected, finite graphs $X=\Gamma \backslash \mathbb{T}_{k}$ )

Let $f: \mathbb{T}_{k} \rightarrow \mathbb{C}$ be a rotation-invariant function with finite support and $\wp_{\Gamma}$ as in Definition 23. Then

$$
\sum_{\lambda_{i} \in \operatorname{Spec}(A)} \widehat{f}\left(\lambda_{i}\right)=f(o)|X|+\sum_{\{\rho\} \in \wp_{\Gamma}} \nu(\rho) \sum_{r \geq 1} H f(r \nu(\rho))
$$

where $H f$ is the horocycle transform in Definition $17, \widehat{f}(\lambda)$ is the spherical transform in Definition 19.

In the next section, we will apply the Selberg Trace Formula to kernels that do not have finite support. So we need a weaker hypothesis on $f$. The Ahumada convergence criterion [1] says that the trace formula holds if

$$
\sum_{n \in \mathbb{Z}}|H f(n)| q^{|n|}<\infty
$$

See also Venkov and Nikitin [46]. Note that we have normalized our horocycle transforms a little differently than these authors.

\section{Clash of the Kernels}

3.1. Kernel \#1. The first kernel is

$$
f_{r}(x, y)= \begin{cases}1, & d(x, y)=r \\ 0, & \text { otherwise }\end{cases}
$$

We have seen that this a natural kernel. Any rotation-invariant, finitely supported function $f: \mathbb{T}_{k} \rightarrow \mathbb{C}$ is a finite linear combination of the $f_{r}$ 's.

Many authors have considered these kernels. We saw in formula (2.8) that they give rise to the Hecke operators $\Theta_{r}$ defined by formula (2.8). The generating function for the Hecke operators is

$$
\sum_{m \geq 0} \Theta_{m} u^{m}=\left(1-u^{2}\right)\left(1-u \Theta_{1}+q u^{2}\right)^{-1} .
$$

Brooks [7] and Quenell [33] use kernel \#1 and the pre-trace formula to bound the diameter, $D$, of a finite $k$-regular graph $X$ with a number depending on the second largest eigenvalue of $A$ in absolute value.

Definition 26. The diameter, $D$, of $X$ is $\max _{x, y \in X} d(x, y)$, where $d(x, y)$ is the length of the shortest path joining $x$ and $y$.

DEFinition 27. The second largest eigenvalue, in absolute value, of the adjacency matrix $\mathbf{A}$ of $\mathbf{X}$ is

$$
\mu=\max \{\lambda \in \operatorname{Spec}(A)|| \lambda \mid \neq k\} .
$$

For example, Quenell [33] shows that

$$
D \leq \frac{\cosh ^{-1}(|X|-1)}{\cosh ^{-1}\left(\frac{k}{\mu}\right)}+1 .
$$

The spherical transform of $f_{r}(x)=f_{r}(d(o, x))$, where $o=$ origin of $\mathbb{T}_{k}$, is

$$
\widehat{f}_{r}(\lambda)=\left\{\begin{array}{ll}
1, & r=0 \\
k(k-1)^{r-1} h_{s}(r), & r>0
\end{array}\right\}
$$


Using Equation (2.1), the horocycle transform is

$$
H f_{r}(m)=\delta_{r}(|m|)+(q-1) \sum_{j=1}^{\infty} q^{j-1} \delta_{r}(|m|+2 j) .
$$

Thus, the Selberg trace formula says $\sum_{\lambda \in \operatorname{Spec}(A)} \widehat{f}_{r}(\lambda)$ equals

$$
f_{r}(o)|X|+\sum_{\{\rho\} \in \wp_{\Gamma}} \nu(\rho) \sum_{e \geq 1}\left(\delta_{r}(e \nu(\rho))+(q-1) \sum_{j=1}^{\infty} q^{j-1} \delta_{r}(e \nu(\rho)+2 j)\right) .
$$

For $r>0$ we can rewrite the trace formula using the $N_{m}$ from Definition 5 as

$$
k(k-1)^{r-1} \sum_{\lambda \in \operatorname{Spec}(A)} h_{\lambda}(r)=\sum_{n \geq g} N_{n}\left(\delta_{r}(n)+(q-1) \sum_{j=1}^{\left[\frac{n}{2}\right]} q^{j-1} \delta_{r}(n+2 j)\right) .
$$

Here $g=$ girth of $X$. This becomes (again for $r>0$ )

$$
k(k-1)^{r-1} \sum_{\lambda \in \operatorname{Spec}(A)} h_{\lambda}(r)=N_{r}+(q-1)\left\{N_{r-2}+q N_{r-4}+\cdots\right\} .
$$

Let us see what happens for various small values of $r$. First note that

$$
\begin{aligned}
& h_{\lambda}(1)=\frac{\lambda}{k}, \quad h_{\lambda}(2)=\frac{\lambda^{2}-k}{k(k-1)}, \quad h_{\lambda}(3)=\frac{\lambda^{3}-(2 k-1) \lambda}{k(k-1)^{2}}, \\
& h_{\lambda}(4)=\frac{\lambda^{4}-(3 k-2) \lambda^{2}+k(k-1)}{k(k-1)^{3}} .
\end{aligned}
$$

Plug $r=1,2$ into Equation (3.1) to see that if $n=|X|,\left\{\lambda_{1}, \ldots, \lambda_{n}\right\}=\operatorname{Spec}(A)$,

$$
\sum_{i=1}^{n} \lambda_{i}=0
$$

$$
\sum_{i=1}^{n} \lambda_{i}^{2}=k n=2|E|, \quad \text { where }|E| \text { is the number of edges in } X \text {. }
$$

These are well known facts. Biggs [5] p. 12 shows

$$
\sum_{i=1}^{n} \lambda_{i}^{l}=\#\{\text { closed paths } C \text { of length } l \text { in } X\} .
$$

Here backtracking and tails are allowed in the paths. Note that a prime path $C$ has no backtracking or tails and $C \neq D^{m}, m>1$.

Suppose $r=g=$ girth $X$. Then equation (3.1) implies

$$
k(k-1)^{g-1} \sum_{i=1}^{n} h_{\lambda_{i}}(g)=g \pi(g)=N_{g} .
$$

One should compare equations (3.5) and (3.6) for their counts. The first counts all closed paths while the second only counts prime paths. 
We need to recall an elementary result from Bollobás [6], (p.68) relating girth and the number of vertices of a graph. Suppose $X$ is a finite graph such that girth $X \geq 3$ and the minimum degree $k \geq 3$. Then, if $n=|X|$,

$$
n \geq \begin{cases}1+\frac{k}{k-2}\left[(k-1)^{\frac{g-1}{2}}-1\right], & \text { if } g \text { is odd, } \\ \frac{2}{k-2}\left[(k-1)^{\frac{g}{2}}-1\right], & \text { if } g \text { is even. }\end{cases}
$$

Then equations (3.4) and (3.7) give us:

Corollary 3. Let $X$ be a finite, connected, $k$-regular, non-bipartite graph such that girth $X \geq 5$ and $k \geq 3$. Then if $\mu$ is the $2 n d$ largest eigenvalue of $A$ in absolute value from Definition 27 , we have $\mu \geq \sqrt{q}$.

Compare the inequality with formula (3.17) below. We omit the proof as it does not really use any non-trivial implications of the Selberg Trace Formula. Solé [35] obtains a stronger result using properties of the spherical functions $h_{\lambda}(n)$ (aka Geronimus polynomials). See also Li and Solé [26].

Beginning with Wigner [48], there has been much thought given to the distribution of the spectrum of a large symmetric real matrix. When the matrix is the adjacency matrix of a graph of large girth, the main result belongs to McKay [27]. Here we give a proof due to Nagoshi $[\mathbf{3 0}],[\mathbf{3 1}]$ which uses the Selberg trace formula for kernel \#1 and the Weyl equidistribution theorem. See also Chung et al [10], Mehta [28], and Sunada [39].

Before stating McKay's result, we need to recall Weyl's equidistribution criterion (see Weyl [47], 1916, or Iwaniec and Kowalski [23], Chapter 21).

Definition 28. A sequence $\left\{x_{n}\right\}$ in an interval $I$ on the real line is said to be equidistributed with respect to a measure $d \mu$ iff for every open set $B$ in interval $I$

$$
\lim _{N \rightarrow \infty} \frac{1}{N}\left|\left\{n \leq N \quad \mid x_{n} \in B\right\}\right|=\mu(B) .
$$

This equidistribution property is equivalent to the statement that for any continuous function with compact support $f$ on $I$ :

$$
\lim _{N \rightarrow \infty} \frac{1}{N} \sum_{n \leq N} f\left(x_{n}\right)=\int_{I} f(x) d \mu(x) .
$$

Definition 29. The Plancherel measure $d \mu_{q}$ on $[-2 \sqrt{q}, 2 \sqrt{q}]$ is given by

$$
d \mu_{q}=\frac{q+1}{2 \pi} \frac{\sqrt{4 q-\lambda^{2}}}{(q+1)^{2}-\lambda^{2}} d \lambda .
$$

The measure $d \mu_{q}$ is that of the Plancherel theorem, stated in formula (2.7), as can be shown by the methods used later in the section on the heat kernel.

Theorem 4. (McKay [27]) Let $\left\{X_{m}\right\}_{m>1}$ be a sequence of $(q+1)$-regular graphs such that for each $r>0$ we have $\lim _{n \rightarrow \infty} \frac{N_{r}}{\left|X_{n}\right|}=0$, where $N_{r}$ is from Definition 5. Then, if $A_{X_{m}}=$ adjacency operator of $X_{m}$, the spectrum of $A_{X_{m}}$ becomes equidistributed with respect to the measure $d \mu_{q}$ on $[-2 \sqrt{q}, 2 \sqrt{q}]$, from Definition 
29, as $n \rightarrow \infty$. More explicitly, this means that if $[\alpha, \beta] \subset[-2 \sqrt{q}, 2 \sqrt{q}]$,

$$
\lim _{m \rightarrow \infty} \frac{\#\left\{\lambda \in \operatorname{Spec}\left(A_{X_{m}}\right) \mid \alpha \leq \lambda \leq \beta\right\}}{\left|X_{m}\right|}=\frac{q+1}{2 \pi} \int_{\alpha}^{\beta} \frac{\sqrt{4 q-\lambda^{2}}}{(q+1)^{2}-\lambda^{2}} d \lambda .
$$

Proof. It suffices to prove formula (3.8) for functions $f$ in an orthogonal basis $\mathcal{B}$ for the space $L^{2}\left(I, d \mu_{q}\right)$, where $I=[-2 \sqrt{q}, 2 \sqrt{q}]$. Such a basis comes from the spherical functions $h_{\lambda}(n)$ on the $k$-regular tree

$$
\mathcal{B}=\left\{\phi_{n}(\lambda)=h_{\lambda}(n)\right\} .
$$

Here $h_{\lambda}(n)$ denotes the spherical function on the $k$-regular tree corresponding to the eigenvalue $\lambda$ of the adjacency operator at a distance $n$ from the origin in the tree. Now we view $h_{\lambda}(n)$ as a function of $\lambda$ holding $n$ fixed. Recall formula (1.2). The spherical function is also a Geronimus polynomial $G_{n}$ as in formula (2.5). See Solé [35] for more information.

Thus our goal is to prove for $I=[-2 \sqrt{q}, 2 \sqrt{q}]$,

$$
\lim _{n \rightarrow \infty} \frac{1}{\left|X_{n}\right|} \sum_{\lambda \in \operatorname{Spec}\left(A_{X_{n}}\right)} h_{\lambda}(r)=\int_{I} h_{\lambda}(r) d \mu_{q}(\lambda)=\left\{\begin{array}{ll}
1, & r=0 \\
0, & r \neq 0 .
\end{array} .\right.
$$

The right hand side of this formula is $\mu_{q}(I)$ for $r=0$ and 0 for $r>0$.

Our formula (3.1) for $r=0$ says, (using the Plancherel theorem (2.7, with some computation which is carried out in more detail for the heat kernel),

$$
1=\frac{1}{\left|X_{n}\right|} \sum_{\lambda \in \operatorname{Spec}\left(A_{X_{n}}\right)} \widehat{f_{0}(\lambda)}=f_{0}(0)=\frac{q+1}{2 \pi} \int_{-2 \sqrt{q}}^{2 \sqrt{q}} \frac{\sqrt{4 q-x^{2}}}{(q+1)^{2}-x^{2}} d x .
$$

Here we note that $r=0$ is always less than the girth of $X_{n}$ so that the right hand side of the trace formula has only one term in this case (the term corresponding to the conjugacy class of the identity).

If $r>0$, must show that we obtain the desired limit

$$
\frac{1}{\left|X_{n}\right|} \sum_{\lambda \in \operatorname{Spec}\left(A_{X_{n}}\right)} \widehat{f_{r}(\lambda)} \rightarrow 0, \text { as } n \rightarrow \infty .
$$

Formula (3.10) follows from formula (3.2) as the right hand side is

$$
\frac{1}{\left|X_{n}\right|} \sum_{\lambda \in \operatorname{Spec}\left(A_{X_{n}}\right)} h_{\lambda}(r)=\frac{1}{\left|X_{n}\right|} \frac{1}{(q+1) q^{r-1}}\left\{N_{r}+(q-1)\left(N_{r-2}+q N_{r-4}+\cdots\right)\right\} \text {. }
$$

Thus the theorem is proved.

If one graphs the limiting densities in McKay's theorem for increasing values of $q$, one begins to see a semicircle. See McKay [27]. Other related references are Chung et al [10], Nagoshi [31], and Sunada [39].

If we let

$$
\phi_{m}(\lambda)= \begin{cases}\frac{q_{m}+1}{2 \pi} \frac{\sqrt{4 q_{m}-\lambda^{2}}}{\left(q_{m}+1\right)^{2}-\lambda^{2}}, & \text { if }|\lambda| \leq 2 \sqrt{q_{m}} \\ 0, & \text { otherwise }\end{cases}
$$


and set $x=\frac{\lambda}{2 \sqrt{q_{m}}}$, then $|x| \leq 1$ and

$$
\frac{\left(q_{m}+1\right)}{2 \pi} \frac{\sqrt{4 q_{m}-\lambda^{2}}}{\left(q_{m}+1\right)^{2}-\lambda^{2}} d \lambda=\frac{2\left(q_{m}^{2}+q_{m}\right) \sqrt{1-x^{2}}}{\pi\left(q_{m}^{2}+2 q_{m}\left(1-2 x^{2}\right)+1\right)} d x
$$

which approaches $\frac{2}{\pi} \sqrt{1-x^{2}} d x$, as $m \rightarrow \infty$.

3.2. Kernel \#2 The Ubiquitous Heat Kernel. For (continuous) time $t>0$, we define the heat kernel by $u_{t}(x)=u_{t}(d(x, o))$, o $=$ origin of $\mathbb{T}_{k}$, with spherical transform,

$$
\widehat{u_{t}}(\lambda)=e^{\omega t}, \omega=\frac{q^{s}+q^{1-s}-(q+1)}{q+1}=\frac{\lambda-k}{k} .
$$

Here, $\omega$ is an eigenvalue of $\frac{1}{k} \widetilde{A}-I=\widetilde{\Delta}$.

This kernel has been considered by many authors (see Bednarchak [4], Chung and Yau [11], Cowling, Meda, and Setti [13]). Some of the motivation is the importance of the classical heat kernel in differential geometry. Chung and Yau [11] use the heat kernel to get an upper bound on the number of spanning trees of $X$.

Some authors consider a different heat kernel with discrete rather than continuous time. See Grigor'yan [18] and Urakawa [44] and [45]. Thus, for a $k$-regular graph $X$, they consider a kernel for the operator $M_{m}=\left(\frac{1}{k} A\right)^{m}$, where $m=$ time. The trace is $\sum_{i=1}^{n}\left(\frac{\lambda_{i}}{k}\right)^{m}$; however, we will not consider the discrete time heat kernel here.

What is the heat equation on $\mathbb{T}_{k}$ ? For us, it is

$$
\left\{\begin{array}{l}
\frac{\partial}{\partial t} y_{t}(x)=\widetilde{\Delta} y_{t}(x), \widetilde{\Delta}=\frac{1}{k} \widetilde{A}-I, \\
y_{0}(x)=f(x) ; \text { rotation-invariant. }
\end{array}\right.
$$

To solve Equation (3.11), take the spherical transform

$$
\left\{\begin{array}{l}
\widehat{\frac{\partial}{\partial t} y_{t}}(\lambda)=\widehat{\widetilde{\Delta} y_{t}}(\lambda), \lambda \in \operatorname{Spec}(\widetilde{\Delta}) \Rightarrow \lambda=\bar{\lambda}, \\
\widehat{y_{0}}(\lambda)=\widehat{f}(\lambda) .
\end{array}\right.
$$

The spherical transform changes $\widetilde{A}$ or $\widetilde{\Delta}=(\widetilde{A}-k I)$ to a multiplication operator:

$$
\widehat{\widetilde{A}} f(s)=\lambda(s) \widehat{f}(s), \lambda(s)=q^{s}+q^{1-s} .
$$

To see this, use Proposition 1 to obtain

$$
\begin{aligned}
\widehat{\widetilde{\Delta} y_{t}}(\lambda) & =\sum_{x \in \mathbb{T}_{k}} \widetilde{\Delta} y_{t}(x) \overline{h_{\lambda}(x)}=\left(\widetilde{\Delta} y_{t}, h_{\lambda}\right)_{\mathbb{T}_{k}}=\left(y_{t}, \widetilde{\Delta} h_{\lambda}\right)_{\mathbb{T}_{k}} \\
& =\bar{\omega}\left(y_{t}, h_{\lambda}\right)_{\mathbb{T}_{k}}=\omega \widehat{y_{t}}(\lambda), \text { since } \omega \text { is real. }
\end{aligned}
$$

Now (3.12) implies $\frac{\partial}{\partial t} \widehat{y}_{t}(\lambda)=\omega \widehat{y_{t}}(\lambda), \widehat{y_{0}}(\lambda)=\widehat{f}(\lambda)$, an ODE with solution $\widehat{y_{t}}(\lambda)=\widehat{f}(\lambda) e^{\omega t}, \omega=\frac{\lambda}{k}-1=\frac{q^{s}+q^{1-s}}{q+1}-1 \in \operatorname{Spec}(\widetilde{\Delta})$. By the convolution properties (see Proposition 2) and the Plancherel theorem for spherical transforms (see formula (2.7)), we have $y_{t}=f * u_{t}$ where $\widehat{u_{t}}(\lambda)=e^{\omega t}$.

So the heat kernel is the fundamental solution of the heat equation on $\mathbb{T}_{k}$. This makes it the analogue of the heat kernel on $\mathbb{R}^{n}$ or the Poincaré upper half plane 
$\mathbb{H}$ in Terras [40]. We will leave open the question of finding an analogue of the central limit theorem on $\mathbb{H}$ as described in Terras [40].

Next, we compute a formula for the heat kernel $u_{t}$ and its horocycle transform $H u_{t}$. To find $H u_{t}$, use Corollary 1 to see that if $s=\frac{1}{2}+i v, \widehat{u_{t}}(s)=e^{\omega t}$, $\omega=\frac{q^{s}+q^{1-s}}{q+1}-1$, and $z=q^{s-\frac{1}{2}}$, we have

$$
\begin{aligned}
H u_{t}(n) q^{\frac{|n|}{2}} & =\frac{1}{2 \pi i} \int_{|z|=1} \widehat{u_{t}}(s) z^{-n} \frac{d z}{z} \\
\left(\text { Set } z=e^{i v \log q}\right) & =\frac{e^{-t} \log q}{2 \pi} \int_{-\frac{\pi}{\log q}}^{\frac{\pi}{\log q}} e^{\frac{q^{s}+q^{1-s}}{q+1} t} e^{-i n v \log q} d v \\
& =\frac{e^{-t} \log q}{2 \pi} \int_{-\frac{\pi}{\log q}}^{\log ^{\frac{2 t \sqrt{q}}{q+1}} \cos (v \log q)} \cos (n v \log q) d v \\
(\text { Set } w=v \log q) & =\frac{e^{-t}}{2 \pi} \int_{-\pi}^{\pi} e^{\frac{2 t \sqrt{q}}{q+1} \cos (w)} \cos (n w) d w .
\end{aligned}
$$

Now we use a formula from Gradshteyn and Ryzhik [17], p. 487 to see that the horocycle transform of the heat kernel is

$$
H u_{t}(n)=q^{-\frac{|n|}{2}} e^{-t} I_{n}\left(\frac{2 t \sqrt{q}}{q+1}\right),
$$

where $I_{n}(z)$ denotes the I-Bessel function (see formula (1.5)). Expansion in series of Bessel functions is a staple in the undergraduate course solving boundary value problems of mathematical physics. For more information, see Courant and Hilbert [12], Lebedev [25], Erdélyi et al [15], or Arfken [2].

To compute $u_{t}(d(x, o))$, we need the Plancherel theorem which was formula (2.7). Chung and Yau [11] obtain the same result by a different method. But first we require a few formulas involving the Harish-Chandra $c$-function. If $\lambda=q^{s}+q^{1-s}$, $s=\frac{1}{2}+i b$, then $\frac{\lambda}{\sqrt{q}}=2 \cos (b \log q)$. Now, $c(s)=\frac{q^{s-1}-q^{1-s}}{(q+1)\left(q^{s-1}-q^{-s}\right)}$ and so

$$
\begin{aligned}
(q+1)^{2}\left|c\left(\frac{1}{2}+i b\right)\right|^{2} & =\frac{q^{-\frac{1}{2}+i b}-q^{\frac{1}{2}-i b}}{q^{-\frac{1}{2}+i b}-q^{-\frac{1}{2}-i b}} \cdot \frac{q^{-\frac{1}{2}-i b}-q^{\frac{1}{2}+i b}}{q^{-\frac{1}{2}-i b}-q^{-\frac{1}{2}+i b}} \\
& =\frac{q^{-1}-q^{2 i b}-q^{-2 i b}+q}{2 q^{-1}-q^{-1+2 i b}-q^{-1-2 i b}} \\
& =\frac{q+\frac{1}{q}-2 \cos (2 b \log q)}{q^{-1}(2-2 \cos (2 b \log q))} .
\end{aligned}
$$

It follows that

$$
q^{-1}(q+1)^{2}\left|c\left(\frac{1}{2}+i b\right)\right|^{2}=\frac{q+\frac{1}{q}-2+4 \sin ^{2}(b \log q)}{4 \sin ^{2}(b \log q)} .
$$

Now, from $\omega(s)=\frac{q^{s}+q^{1-s}}{q+1}-1, s=\frac{1}{2}+i b$, and $k=q+1$, we can write $e^{t \omega(s)}$ $=e^{-t} e^{\frac{t}{k} 2 \sqrt{q} \cos (b \log q)}$ and the Plancherel theorem (formula (2.7)) plus the formula 
for the Harish-Chandra c-function (formula (2.3)) imply

$$
\begin{aligned}
u_{t}(y) & =\int_{0}^{\frac{\pi}{\log q}} \widehat{u_{t}}\left(\frac{1}{2}+i b\right) h_{\frac{1}{2}+i b}(y) \frac{q \log q}{2 \pi(q+1)\left|c\left(\frac{1}{2}+i b\right)\right|^{2}} d b \\
& =\frac{2 q(q+1) e^{-t}}{\pi} \int_{0}^{\pi} e^{\frac{2 t \sqrt{q}}{k} \cos (x)} h_{\frac{1}{2}+i \frac{x}{\log q}}(y) \frac{\sin ^{2}(x)}{(q+1)^{2}-4 q+4 q \sin ^{2}(x)} d x .
\end{aligned}
$$

Then, by setting $x=b \log q$, we have

$$
u_{t}(y)=\frac{2 q k e^{-t}}{\pi} \int_{0}^{\pi} e^{\frac{2 t \sqrt{q}}{k} \cos (x)} h_{\frac{1}{2}+i \frac{x}{\log q}}(y) \frac{\sin ^{2}(x)}{k^{2}-4 q \cos ^{2}(x)} d x
$$

Claim 1. When $d>0$,

$$
\begin{aligned}
h_{\frac{1}{2}+i b}(y) & =h_{\frac{1}{2}+i b}(d(y, o))=h_{\frac{1}{2}+i b}(d) \\
& =\frac{q^{-\frac{d}{2}}}{q+1}\left(\frac{q \sin ((d+1) x)-\sin ((d-1) x)}{\sin (x)}\right) \text {, if } x=b \log q .
\end{aligned}
$$

Proof. Writing $z=q^{i b}$ and letting $x=b \log q$, we have from formula (2.4)

$$
\begin{aligned}
(q+1) q^{\frac{d}{2}} h_{\frac{1}{2}+i b}(d) & =\frac{q q^{-i b d}\left(q^{i b(2+2 d)}-1\right)-q^{i b d}\left(1-q^{i b(2-2 d)}\right)}{q^{2 i b}-1} \\
& =\frac{q\left(q^{i b(d+1)}-q^{-i b(d+1)}\right)-\left(q^{i b(d-1)}-q^{-i b(d-1)}\right)}{q^{i b}-q^{-i b}} \\
& =\frac{q \sin ((d+1) x)-\sin ((d-1) x)}{\sin (x)} .
\end{aligned}
$$

Thus, for $d>0$, our claim gives us the formula for the heat kernel except at the origin

$$
u_{t}(y)=\frac{2 q^{1-\frac{d}{2}} e^{-t}}{\pi} \int_{0}^{t} e^{\frac{2 t \sqrt{q}}{k} \cos (x)} \frac{\sin (x)(q \sin ((d+1) x)-\sin ((d-1) x))}{k^{2}-4 q \cos ^{2}(x)} d x .
$$

When $d=0$, we have $h_{\frac{1}{2}+i b}(0)=1$, and then we obtain the formula for the heat kernel at the origin

$$
u_{t}(o)=\frac{2 q k e^{-t}}{\pi} \int_{0}^{\pi} e^{\frac{2 t \sqrt{q}}{k} \cos (x)} \frac{\sin ^{2}(x)}{k^{2}-4 q \cos ^{2}(x)} d x .
$$

These two formulas for $u_{t}$ agree with those in Chung and Yau [11].

Plugging the heat kernel $u_{t}$ into the trace formula yields (using formulas (3.13) and (3.16))

$$
\sum_{i=1}^{n} e^{\omega_{i} t}=n u_{t}(o)+e^{-t} \sum_{\{\rho\} \in \wp_{\Gamma}} \nu(\rho) \sum_{e \geq 1} q^{-\frac{e \nu(\rho)}{2}} I_{e \nu(\rho)}\left(\frac{2 t \sqrt{q}}{q+1}\right) .
$$

Here $n=|X|, \omega_{i}=\frac{\lambda_{i}}{k}-1$, where $\operatorname{Spec}(A)=\left\{\lambda_{1}, \ldots, \lambda_{n}\right\}$, and $k=q+1$. By factoring out $e^{-t}$ from both sides, we see 


$$
\begin{aligned}
\sum_{i=1}^{n} e^{\frac{\lambda_{i} t}{k}}= & \frac{2 q k n}{\pi} \int_{0}^{\pi} e^{\frac{2 t \sqrt{q}}{k} \cos (x)} \frac{\sin ^{2}(x)}{k^{2}-4 q \cos ^{2}(x)} d x \\
& +\sum_{\{\rho\} \in \wp \Gamma} \nu(\rho) \sum_{e \geq 1} q^{-\frac{e \nu(\rho)}{2}} I_{e \nu(\rho)}\left(\frac{2 t \sqrt{q}}{q+1}\right) .
\end{aligned}
$$

Recall that $\rho$ is a primitive hyperbolic element of $\mathbb{T}_{k}$ that corresponds to a prime path $C$ in the graph $X$. Therefore, the sum on the right-hand side can written in the following form:

$$
\begin{aligned}
\sum_{\{\rho\} \in \wp_{\Gamma}} \nu(\rho) \sum_{e \geq 1} q^{-\frac{\nu\left(\rho^{e}\right)}{2}} I_{\nu\left(\rho^{e}\right)}\left(\frac{2 t \sqrt{q}}{q+1}\right) & =\sum_{C} \sum_{e \geq 1} q^{-\frac{v\left(C^{e}\right)}{2}} I_{\nu\left(C^{e}\right)}\left(\frac{2 t \sqrt{q}}{q+1}\right) \\
& =\sum_{n \geq 1} N_{n} q^{-\frac{n}{2}} I_{n}\left(\frac{2 t \sqrt{q}}{q+1}\right),
\end{aligned}
$$

where $N_{n}$ is from Definition 5. This implies formula (1.4) of the introduction.

We will see using kernels \#3 and \#6 that

$$
\begin{aligned}
& N_{m} \sim q^{m}, \quad \text { as } m \rightarrow \infty, \quad \text { if } X \text { is non - bipartite; } \\
& N_{2 m} \sim 2 q^{2 m}, \quad \text { as } m \rightarrow \infty, \quad \text { if } X \text { is bipartite. }
\end{aligned}
$$

Thus, using formula (1.5) we see that the series converges:

$$
\begin{aligned}
\sum_{\{\rho\} \in \wp_{\Gamma}} \nu(\rho) \sum_{e \geq 1} q^{-\frac{e \nu(\rho)}{2}} I_{e \nu(p)}\left(\frac{2 t \sqrt{q}}{q+1}\right) & \ll \sum_{n=-\infty}^{\infty} q^{\frac{n}{2}} I_{n}\left(\frac{2 t \sqrt{q}}{q+1}\right) \\
& =e^{\frac{t \sqrt{q}}{q+1}\left(\sqrt{q}+\frac{1}{\sqrt{q}}\right)} .
\end{aligned}
$$

3.3. Kernel \#3 Kernel used to prove Ihara-Zeta function of $X$ is reciprocal of a polynomial. We discussed this kernel in [41] so we will be brief. The kernel is defined by its horocycle transform

$$
H \alpha_{u}(n)= \begin{cases}u^{|n|-1}, & n \neq 0 \\ 0, & n=0\end{cases}
$$

The Ahumada convergence criterion $[\mathbf{1}]$ says if $|u|<\frac{1}{q}$,

$$
\sum\left|H \alpha_{u}(n)\right| q^{|n|} \leq \frac{1}{|u|} \sum|u|^{n} q^{|n|}=\frac{1}{|u|} \frac{1}{1-q|u|} .
$$

Setting $\lambda=q^{s}+q^{1-s}, \widehat{\alpha_{u}}(\lambda)=\frac{d}{d u} \log \left(\frac{1}{1-\lambda u+q u^{2}}\right)$. One has $\alpha_{u}(0)=\frac{(1-q) u}{1-u^{2}}$ and $\alpha_{u}(d)=\alpha_{u}(d(x, o))=\frac{u^{d-1}\left(1-q u^{2}\right)}{1-u^{2}}$, for $d>0$. Then the Selberg trace formula for the kernel $\alpha_{u}$ says:

$$
\sum_{i=1}^{n} \frac{d}{d u} \log \left(\frac{1}{1-\lambda_{i} u+q u^{2}}\right)=\frac{(1-q) u}{1-u^{2}}|X|+\sum_{\{\rho\}} \nu(\rho) \sum_{r \geq 1} u^{r \nu(\rho)-1}
$$

which implies the theorem below. 
Theorem 5. (Ihara) Given $X$, a $(q+1)$-regular, connected, finite graph

$$
\zeta_{X}^{-1}(u)=\left(1-u^{2}\right)^{r-1} \operatorname{det}\left(I-A u+q u^{2} I\right),
$$

where $r-1=\frac{|X|(q-1)}{2}=|E|-|V|, E$ and $V$ are the edges and vertices of $X$, respectively, and $r$ is the rank of the fundamental group of $X$.

From this theorem, one can deduce the following corollary. See the last page of Terras $[\mathbf{4 1}]$.

Corollary 4. For a graph $X$ satisfying the hypotheses of the preceding theorem, the complexity of a graph, $\kappa(X)$, which is the number of spanning trees of $X$, is

$$
\lim _{u \rightarrow 1} \frac{-1}{(r-1) 2^{r}} \cdot \frac{1}{(1-u)^{r} \zeta_{X}(u)} .
$$

Formula (1.6) for $N_{m}$, which was given in the introduction, is also implied by Theorem 5. To see this note that

$$
\begin{aligned}
\operatorname{det}\left(I-A u+q u^{2} I\right) & =\prod_{i=1}^{n}\left(1-\lambda_{i} u+q u^{2}\right) \\
& =\prod_{i=1}^{n}\left(1-\alpha_{\lambda_{i}} u\right)\left(1-\beta_{\lambda_{i}} u\right)
\end{aligned}
$$

where $\left\{\lambda_{i}\right\}_{i=1}^{n}=\operatorname{Spec}(A)$.

Then apply $u \frac{d}{d u}$ to the Ihara formula and see that

$$
\begin{aligned}
u \frac{d}{d u} \log \zeta_{X}(u) & =\sum_{m \geq 1} N_{m} u^{m} \\
& =\sum_{m \geq 1} u^{m}\left\{(r-1)\left(1+(-1)^{m}\right)+\sum_{i=1}^{n}\left(\alpha_{\lambda_{i}}^{m}+\beta_{\lambda_{i}}^{m}\right)\right\},
\end{aligned}
$$

where $N_{m}$ is from Definition 5. Formula (1.6) is then clear.

One interesting fact about the Ihara zeta function is that the analogue of the Riemann hypothesis is equivalent to the graph being Ramanujan, which means that $\mu$ from Definition 27, i.e., the second largest eigenvalue of $A$ in absolute value, satisfies

$$
\mu \leq 2 \sqrt{q}
$$

Ramanujan graphs are of interest in computer science because they lead to efficient communication networks. See Terras $[\mathbf{4 1}]$ for more information.

The Ihara theorem holds for irregular graphs thanks to the work of Bass [3] and Hashimoto [19]. One simply replaces $q u^{2} I$ with $u^{2} Q$ where $Q$ is the diagonal matrix whose $j^{\text {th }}$ entry is $q_{j}$, where $q_{j}+1$ is the degree of the $j^{\text {th }}$ vertex of $X$. See Stark and Terras [36] for more information. You can view the logarithmic derivative of this result as a replacement for the Selberg trace formula for irregular graphs. See Mizuno and Sato [29].

M. Horton $[\mathbf{2 0}]$ has shown that the girth of $X$ is the smallest exponent $i$ with $c_{i} \neq 0$ in the polynomial $\zeta_{X}^{-1}(u)=1+c_{1} u+c_{2} u^{2}+\ldots+c_{2|E|} u^{2|E|}$. Moreover,

$$
c_{g}=-\frac{N_{g}}{g}=-\pi(g) \text {. }
$$


Horton [21] has also shown that for a graph $X$ there exists a sequence of graphs $\left\{X_{n}\right\}_{n \geq 1}$ such that $X$ is a subgraph of $X_{n}$, the $X_{n}$ are distinct, and $\lim _{n \rightarrow \infty} \zeta_{X_{n}}(u)=$ $\zeta_{X}(u)$ for each $u$ such that $|u|<1$.

D. Newland [32] has studied the level spacings of zeros of $\zeta_{X}(u)^{-1}$ and compared them to that of the zeros of other zeta functions such as Riemann's. Though many zeta functions have level spacings in the Gaussian Unitary Ensemble (GUE), since the adjacency matrix involved in $\zeta_{X}(u)$ is a real, symmetric matrix, its level spacings are more closely related to those of the spectrum of the adjacency matrix of $X$. This will be the distribution of the Gaussian Orthogonal Ensemble (GOE) for randomly generated, $k$-regular graphs. For certain arithmetically defined Cayley graphs (the Euclidean graphs in Terras [41]), however, it appears that the level spacings are those of Poisson random variables. This is similar to what happens for the explicit examples of function fields zeta functions in Katz and Sarnak [24].

\subsection{Kernel \#4.}

$$
\widehat{f_{\alpha, \beta}}(\lambda)=\delta_{[\alpha, \beta]}(\lambda)= \begin{cases}1, & \alpha \leq \lambda \leq \beta \\ 0, & \text { otherwise }\end{cases}
$$

This kernel is a natural one to think about. It appears in Mizuno and Sato [29] for bi-regular bipartite graphs. The problem is that the Ahumada convergence condition fails for this kernel, as we shall see.

The Plancherel theorem implies (setting $w=2 \sqrt{q} \cos x$ in the kernel \#4 analog of formula (3.14)) that

$$
\begin{aligned}
& f_{\alpha, \beta}(x)=\frac{q+1}{2 \pi} \int_{\alpha}^{\beta} h_{\lambda}(x) \frac{\sqrt{4 q-\lambda^{2}}}{(q+1)^{2}-\lambda^{2}} d \lambda, \quad \text { if } d(x, o)>0 \\
& f_{\alpha, \beta}(o)=\frac{q+1}{2 \pi} \int_{\alpha}^{\beta} \frac{\sqrt{4 q-\lambda^{2}}}{(q+1)^{2}-\lambda^{2}} d \lambda .
\end{aligned}
$$

The horocycle transform here is

$$
H f_{\alpha, \beta}(n) q^{\frac{|n|}{2}}=\frac{1}{2 \pi i} \int_{|z|=1} \delta_{[\alpha, \beta]}\left(q^{\frac{1}{2}}\left(z+z^{-1}\right)\right) z^{-n} \frac{d z}{z} .
$$

We have for $z=q^{i v}$,

$$
2 \sqrt{q} \cos (v \log q) \in[\alpha, \beta] \Leftrightarrow \cos (v \log q) \in\left[\frac{\alpha}{2 \sqrt{q}}, \frac{\beta}{2 \sqrt{q}}\right] .
$$

If $-2 \sqrt{q}<\alpha<\beta<2 \sqrt{q}$, and $(\alpha, \beta)$ is an interval on which $\cos ^{-1}(\theta)$ is well defined,

$$
v \log q \in\left[\cos ^{-1}\left(\frac{\alpha}{2 \sqrt{q}}\right), \cos ^{-1}\left(\frac{\beta}{2 \sqrt{q}}\right)\right] .
$$


So letting $z=e^{i v \log q}$, we get $\frac{d z}{z}=i \log q d v$ and

$$
\begin{aligned}
H f_{\alpha, \beta}(n) q^{\frac{|n|}{2}} & =\frac{\log q}{2 \pi} \int_{\frac{\cos ^{-1}\left(\frac{\beta}{2 \sqrt{q}}\right)}{\log q}}^{\frac{\cos ^{-1}\left(\frac{\alpha}{2 \sqrt{q}}\right)}{\log q}} \cos (n v \log q) d v \\
& =\frac{\sin (n v \log q)}{2 \pi n} \mid \begin{array}{l}
\cos ^{-1}\left(\frac{\alpha}{2 \sqrt{q}}\right) / \log q \\
\cos ^{-1}\left(\frac{\beta}{2 \sqrt{q}}\right) / \log q
\end{array} \\
& =\frac{1}{4 \sqrt{q} \pi n}\left(\sqrt{4 q-\alpha^{2}} U_{n-1}\left(\frac{\alpha}{2 \sqrt{q}}\right)-\sqrt{4 q-\beta^{2}} U_{n-1}\left(\frac{\beta}{2 \sqrt{q}}\right)\right),
\end{aligned}
$$

where $U_{n}(x)$ is the Chebyshev polynomial in formula (1.1). Now, $U_{n}(x)$ has the generating function

$$
\sum_{n \geq 0} U_{n}(x) z^{n}=\left(1-2 x z+z^{2}\right)^{-1}
$$

when $|x|<1,|z|<1$.

Does our horocycle transform satisfy the Ahumada condition? That is to say, is $\sum H f_{\alpha, \beta}(n) q^{|n|}<\infty$ ? Note that

$$
\sum_{n \geq 1} q^{|n|} \frac{q^{-\frac{|n|-1}{2}}}{4 \pi|n|} \sqrt{4 q-\beta^{2}} U_{n-1}\left(\frac{\beta}{2 \sqrt{q}}\right)=\frac{\sqrt{4 q-\beta^{2}}}{4 \pi} \sum_{n \geq 1} \frac{q^{\frac{|n|+1}{2}}}{n} U_{n-1}\left(\frac{\beta}{2 \sqrt{q}}\right) .
$$

Since

$$
U_{n}(0)= \begin{cases}0, & \text { if } n \text { is odd, } \\ (-1)^{m}, & \text { if } n=2 m\end{cases}
$$

when $\beta=0$, we have a divergent series.

3.5. Kernel \#5 Resolvent Kernel or Green's Function. The resolvent kernel $G_{w}(d(x, o))$ is defined by

$$
\widehat{G_{w}}(s)=\frac{1}{\lambda(s)-\lambda(w)}
$$

for $\lambda(s)=q^{s}+q^{1-s}=2 \sqrt{q} \cos (\theta \log q), s=\frac{1}{2}+i \theta, \lambda(s) \in[-2 \sqrt{q}, 2 \sqrt{q}]$, and $\lambda(w)=q^{w}+q^{1-w}, \lambda(w) \notin[-2 \sqrt{q}, 2 \sqrt{q}]$. This kernel has appeared in Cartier [8], Chung and Yau [11], with $\lambda(w)=0$, and Figà-Talamanca and Nebbia [16]. The last authors use it to prove the Plancherel theorem and they show

$$
\left(A-\left(q^{w}+q^{1-w}\right) I\right)^{-1} f(x)=\sum_{y \in \mathbb{T}_{k}} \frac{q^{-w d(x, y)}}{q^{-w}-q^{w}} f(y) .
$$

For the Selberg trace formula on the Poincare upper half-plane, the resolvent kernel is used by Elstrodt [14] to study Selberg's zeta function. But he has to look at the difference $G_{a}-G_{b}$ to satisfy a convergence condition. We will not have to do this here.

Let $\eta(n)=-\frac{q^{-w|n|}}{q^{w}-q^{1-w}}$. We can show that this is the horocycle transform of the resolvent kernel; i.e., $\eta(n)=H G_{w}(n)$. For this, it suffices (using Lemma 1) to 
show that

$$
\sum_{n \in \mathbb{Z}} \eta(n) q^{\frac{|n|}{2}} q^{i \theta n}=\widehat{G_{w}}(\lambda)
$$

where $\lambda=2 \sqrt{q} \cos (\theta \log q)$.

To prove formula (3.19), note:

$$
\begin{aligned}
-\left(q^{w}-q^{1-w}\right) \sum_{n \in \mathbb{Z}} \eta(n) q^{\frac{|n|}{2}} q^{i \theta n} & =\sum_{n \in \mathbb{Z}} q^{-w|n|+i \theta n+\frac{|n|}{2}} \\
& =1+\frac{q^{-w+i \theta+\frac{1}{2}}}{1-q^{-w+i \theta+\frac{1}{2}}}+\frac{q^{-w-i \theta+\frac{1}{2}}}{1-q^{-w-i \theta+\frac{1}{2}}} \\
& =\frac{1-q^{-2 w+1}}{1+q^{-2 w+1}-2 q^{-w} \sqrt{q} \cos (\theta \log q)} \\
& =-\left(q^{w}-q^{1-w}\right) \frac{1}{\lambda(s)-\lambda(w)} .
\end{aligned}
$$

Thus, $\widehat{f}\left(\frac{1}{2}+i \theta\right)=\widehat{G_{w}}=\frac{1}{\lambda(s)-\lambda(w)}$, as claimed.

Does $\left(H G_{w}\right)(n)$ satisfy the Ahumada condition? Yes, if $\operatorname{Re} w>1$.

Proof. $\sum_{n \in \mathbb{Z}}\left(H G_{w}\right)(n) q^{|n|}<\infty \Leftrightarrow \sum_{n \geq 1}\left(q^{1-w}\right)^{n}<\infty \Leftrightarrow \operatorname{Re} w>1$.

Here

$$
G_{w}(o)=\frac{q+1}{2 \pi} \int_{-2 \sqrt{q}}^{2 \sqrt{q}} \frac{\sqrt{4 q-\lambda^{2}}}{(q+1)^{2}-\lambda^{2}} \frac{1}{\lambda-\left(q^{w}+q^{1-w}\right)} d \lambda,
$$

by Plancherel. So, we can plug this kernel legally into the trace formula and obtain the $N_{m}$ formula (1.8) which was stated in the introduction.

Note that kernel \#5 is closely related to kernel \#3.

\subsection{Kernel \#6 Kernel for which Horocycle Transform is a Delta}

\section{Function. Let}

$$
\left(H g_{n}\right)(m)=\left\{\begin{array}{ll}
1, & m= \pm n, \\
0, & \text { otherwise, }
\end{array} \text { for } n \geq 0 .\right.
$$

Inversion of the horocycle transform (see formula (2.2)) yields

$$
\begin{aligned}
g_{n}(|m|) & =\delta_{n}(|m|)-(q-1) \sum_{j \geq 0} \delta_{n}(|m|+2 j) \\
& =\delta_{n}(|m|)-(q-1) \sum_{j=1}^{\left\lceil\frac{n}{2}\right\rceil} \delta_{n-2 j}(|m|),
\end{aligned}
$$

which implies, for $n>0$

$$
g_{n}(0)=-(q-1) \begin{cases}1, & \text { if } 2 \mid n, \\ 0, & \text { otherwise. }\end{cases}
$$

The spherical transform is, by Lemma 1 , for $z=q^{s-\frac{1}{2}}$

$$
\widehat{g_{n}}(s)=q^{\frac{n}{2}}\left(z^{n}+z^{-n}\right)= \begin{cases}q^{n s}+q^{n(1-s)}, & n>0, \\ 1, & n=0 .\end{cases}
$$


So the trace formula says if $A=$ adjacency operator of $X$ and $n>0$,

$$
\begin{aligned}
& \sum_{\lambda=q^{s}+q^{1-s} \in \operatorname{Spec}(A)}\left(q^{n s}+q^{n(1-s)}\right)=-(q-1)|X| \begin{cases}1, & n \text { even, } \\
0, & n \text { odd }\end{cases} \\
& +\sum_{\{\rho\} \in \wp_{\Gamma}} \nu(\rho) \sum_{r \geq 1} \delta_{n}(r \nu(\rho)) \text {. }
\end{aligned}
$$

Both sides of this formula are finite. This implies the $N_{m}$ formula (1.9) of the introduction. For small values of $n$, this yields the same results as kernel \#1. But this could be a useful kernel to convolve with others.

Conclusion 1. Which kernel won the contest? Which kernel lost? We leave the final decisions to the reader. It appears to us, however, that kernels \#1, \#3, and \#6 are in the lead and kernel \#4 never got out of the gate.

\section{References}

[1] G. Ahumada, Fonctions périodiques et formule des traces de Selberg sur les arbres, C.R. Acad. Sci. Paris Sér. I Math, 305 (1987), no. 709-712.

[2] G. Arfken, Mathematical Methods for Physicists, Academic, New York, 1970.

[3] H. Bass, The Ihara-Selberg zeta function of a tree lattice, Int. J. Math. 3 (1992), no. 6, 717-797.

[4] D. Bednarchak, The heat kernel for regular trees, Cont. Math, 206 (1997), 111-112.

[5] N. Biggs, Algebraic Graph Theory, Cambridge Univ. Press, London, 1974.

[6] B. Bollobás, Graph Theory: An Introductory Course, Springer-Verlag, New York, 1979.

[7] R. Brooks, The spectral geometry of k-regular graphs, J. Anal. Math. 57 (1991), 120-151.

[8] P. Cartier, Harmonic analysis on trees, Harmonic Analysis on Homogeneous Spaces (Proc. Sympos. Pure Math., Vol. 26, Williams Coll., Williamstown, Mass., 1972), American Mathematical Society, Rhode Island, 1973, 419-424.

[9] P. Cartier, Géométrie et analyse sur les arbres, Sém. Bourbaki, 1971/72, n 407, 1-17.

[10] F. Chung, L. Lu, V. Vu, Spectra of random graphs with given expected degrees, Proc. Natl. Acad. Sci., 100, No. 11 (2003), 6313-6318.

[11] F. R. K. Chung and S. -T. Yau, Coverings, heat kernels and spanning trees, Elec. J. Combinatorics, 6 (1999) \#R12.

[12] R. Courant and D. Hilbert, Methods of Mathematical Physics, Vol. I, Wiley-Interscience, New York, 1953.

[13] M. Cowling, S. Meda, and A. Setti, Estimates for functions of the Laplace operator on homogeneous trees, Trans. A.M.S., 352 (2000), 4271-4293.

[14] J. Elstrodt, Die Selbergsche Spurformel für Kompakte Riemannsche Flächen, Jahresbericht d. Deutch. Math.-Verein, 83 (1981), 45-77.

[15] A. Erdélyi et al (Eds.), Higher Transcendental Functions, McGraw-Hill, New York, 1953, Vol. II.

[16] A. Figà-Talamanca and C. Nebbia, Harmonic Analysis and Representation Theory for Groups Acting on Homogeneous Trees, London Mathematical Society Lecture Note Series, vol. 162, Cambridge University Press, Cambridge, 1991.

[17] I. S. Gràdshteyn and I. M. Ryzhik, Table of Integrals, Series and Products, Academic, Orlando, Florida, 1965.

[18] A. Grigor'yan, Heat kernels on manifolds, graphs and fractals, European Cong. Math, I (Barcelona, 2000), 393-406, Prog. Math. 201, Birkhäuser, Basel, 2001.

[19] K. Hashimoto, Zeta functions of finite graphs and representations of p-adic groups, Automorphic Forms and Geometry of Arithmetic Varieties, Advanced Studies in Pure Mathematics, vol. 15, Academic Press, Massachusetts, 1989, 211-280.

[20] M. Horton, Recovering girth from the Ihara zeta function, preprint.

[21] M. Horton, The Ihara zeta function and deleting edges, preprint.

[22] N. Hurt, Mathematical Physics of Quantum Wires and Devices, Kluwer, Dordrecht, 2000.

[23] H. Iwaniec and E. Kowalski, Analytic Number Theory, A.M.S., Providence, 2004. 
[24] N. Katz and P. Sarnak, Zeros zeta functions and symmetry, Bull. Amer. Math. Soc. (N.S.) 36 (1999), no. 1, 1-26.

[25] N. Lebedev, Special Functions and Their Applications, Dover, New York, 1972.

[26] W. Li and P. Solé, Spectra of regular graphs and hypergraphs and orthogonal polynomials, Europ. J. Combinatorics, 17 (1996), 461-477.

[27] B. D. McKay, The expected eigenvalue distribution of a large regular graph, Linear Algebra and Its Applications, 40 (1981), 203-216.

[28] M. L. Mehta, Random Matrices and the Statistical Theory of Energy Levels, Academic, N.Y., 1990.

[29] H. Mizuno and I. Sato, The semicircle law for semi-regular bipartite graphs, J. Comb. Theory, A, 101, (2003), 174-190.

[30] H. Nagoshi, On arithmetic infinite graphs, Proc. Japan Acad., 76, Ser. A (2000), 22-25.

[31] H. Nagoshi, Spectral theory of certain arithmetic graphs, Cont. Math., Vol. 347, Amer. Math. Soc., Providence, 2004, pp. 203-220.

[32] D. Newland, Distribution of the Zeros of the Ihara Zeta Function, preprint.

[33] G. Quenell, Spectral diameter estimates for k-regular graphs, Adv. Math. 106 (1994), no. 1, 122-148.

[34] A. Selberg, Harmonic analysis and discontinuous groups in weakly symmetric Riemannian spaces with applications to Dirichlet series, J. Indian Math. Soc. (N.S.) 20 (1956), 47-87.

[35] P. Solé, The second eigenvalue of regular graphs of given girth, J. Comb. Theory, B, 56 (1992), 239-249.

[36] H. M. Stark and A. A. Terras, Zeta functions of finite graphs and coverings, Adv. Math. 121 (1996), no. 1, 124-165.

[37] H. M. Stark and A. A. Terras, Zeta functions of finite graphs and coverings II, Adv. Math. 154 (2000), no. 1, 132-195.

[38] T. Sunada, Fundamental groups and Laplacians, Geometry and Analysis on Manifolds (Katata/Kyoto, 1987), Lecture Notes in Mathematics, 1339, Springer-Verlag, New York, 1988, 248-277.

[39] T. Sunada, The discrete and the continuous, Sugaku Seminar (Japanese) 40, Nippon Hyouronsha, Tokyo, 2001, 48-51.

[40] A. Terras, Harmonic Analysis on Symmetric Spaces and Applications I, Springer-Verlag, New York, 1985.

[41] A. Terras, Fourier Analysis on Finite Groups and Applications, London Mathematical Society Student Texts, vol. 43, Cambridge University Press, Cambridge, 1999.

[42] A. Terras, Comparison of Selberg's trace formula with its discrete analogues, DIMACS Series in Discrete Math., Vol. 64, Amer. Math. Soc., Providence, 2004, pages 213-225.

[43] A. Terras and D. Wallace, Selberg's Trace Formula on the k-regular tree and Applications, Intl. J. Math. and Math. Sciences, 8 (2003), 501-526.

[44] H. Urakawa, Spectra of discrete and continuous Laplacians on graphs and Riemannian manifolds, Interdisciplinary Information Sciences, Vol. 3, No. 2 (1997), 95-109.

[45] H. Urakawa, Heat kernel and Green kernel comparison theorems for infinite graphs, J. Functional Anal. 146 (1997), 206-235.

[46] A. B. Venkov and A. M. Nikitin, The Selberg trace formula, Ramanujan graphs and some problems in mathematical physics, Algebra i Analiz 5 (1993), no. 3, 1-76 (Russian), translated in St. Petersburg Math. J. 5 (1994), no. 3, 419-484.

[47] H. Weyl, Über die Gleichverteilung von Zahlen mod. Eins, Math. Ann., 77 (1916), 313-352.

[48] E. P. Wigner, Random matrices in physics, S.I.A.M. Review, Vol. 9, No. 1 (1967), 1-23.

Math Department, U.C.S.D., 9500 Gilman Drive, La Jolla, CA 92093-0112 